\title{
Perturbation Theory of Transformed Quantum Fields
}

\section{Paul-Hermann Balduf ${ }^{1}$}

Received: 4 June 2019 / Accepted: 19 August 2020 / Published online: 10 September 2020

(C) The Author(s) 2020

\begin{abstract}
We consider a scalar quantum field $\phi$ with arbitrary polynomial self-interaction in perturbation theory. If the field variable $\phi$ is repaced by a global diffeomorphism $\phi(x)=\rho(x)+a_{1} \rho^{2}(x)+\ldots$, this field $\rho$ obtains infinitely many additional interaction vertices. We propose a systematic way to compute connected amplitudes for theories involving vertices which are able to cancel adjacent edges. Assuming tadpole graphs vanish, we show that the $S$-matrix of $\rho$ coincides with the one of $\phi$ without using path-integral arguments. This result holds even if the underlying field has a propagator of higher than quadratic order in the momentum. The diffeomorphism can be tuned to cancel all contributions of an underlying $\phi^{t}$-type self interaction at one fixed external offshell momentum, rendering $\rho$ a free theory at this momentum. Finally, we mention one way to extend the diffeomorphism to a non-diffeomorphism transformation involving derivatives without spoiling the combinatoric structure of the global diffeomorphism.
\end{abstract}

Keywords Diffeomorphism of quantum fields · Propagator cancellation · Bell polynomials · Diffeomorphism invariance of the S-matrix - Connected perspective

Mathematics Subject Classification (2010) $81 \mathrm{~T} 18$

\section{Introduction}

\subsection{Motivation and Content}

A quantum field theory can be defined via a Lagrangian density $\mathcal{L}$, simply called Lagrangian hereafter. In perturbative computations in this theory, the monomials of

The author thanks Dirk Kreimer and Karen Yeats for helpful discussion.

Paul-Hermann Balduf

paul.balduf@physik.hu-berlin.de

1 Institut für Physik, Humboldt-Universität zu Berlin, Newtonstrasse 15, 12489 Berlin, Germany 
degree $n>2$ in $\mathcal{L}$ correspond to $n$-valent interaction vertices in Feynman diagrams. The monomials of degree two define the propagator of the field. We only consider scalar quantum fields in this paper. A free scalar quantum field theory has no selfinteraction and is defined via the Lagrangian

$$
\mathcal{L}_{\phi}=\frac{1}{2} \partial_{\mu} \phi(x) \partial^{\mu} \phi(x)-\frac{1}{2} m^{2} \phi^{2}(x),
$$

where $m \geq 0$ is the mass of a $\phi$-particle. We allow $m=0$ for the massles theory. $x$ is a point in spacetime which we take to be 4-dimensional Minkowski space for concreteness even if our results do not depend on the dimensionality.

We express the field $\phi(x)$ as a global diffeomorphism

$$
\phi(x)=\sum_{j=0}^{\infty} a_{j} \rho^{j+1}(x), \quad a_{0}=1
$$

of another field $\rho(x)$. The term global stresses that $\left\{a_{j}\right\}_{j \geq 1}$ are constants with respect to spacetime, i.e. the diffeomorphism is the same transformation globally. This is common physics nomenclature (e.g. global vs local gauge symmetries), alternatively one could call (1.2) local since it only involves $\rho(x)$ at that very point $x$ (that is the perspective of [17]). The constraint $a_{0}=1$ means the diffeomorphism is tangent to identity such that the fields $\rho$ and $\phi$ coincide at leading order. Equation 1.2, when applied to the Lagrangian $\mathcal{L}_{\phi}$ of $\phi$, gives rise to a Lagrangian $\mathcal{L}_{\rho}$ of $\rho$ which generally involves monomials of any order. Thus, $\rho$ is an interacting quantum field theory even if the original field $\phi$ was free.

If Eq. 1.2 is applied to a classical field theory before canonical quantization, it amounts to a canonical transformation which leaves the poisson brackets intact [22] and only changes the Lagrangian, thus relating theories with different Lagrangians.

In the framework of the path integral, observables appear invariant under diffeomorphisms as the diffeomorphism can be undone by a redefinition of the integration measure. However, different lines of argument lead to different results [1] which might be due to operator ordering ambiguities [23]. One possibility to resolve these puzzles is to understand field diffeomorphisms order by order in perturbation theory and then, in a later step, extend these results to a statement about the generating functionals of Feynman graphs. This might be a mathematically cleaner way than direct, formal manipulations of field variables within (divergent) generating functionals. This argument is inspired by the recent proof [13] that the Legendre transform - which relates the generating functionals of connected Feynman graphs to that of 1PI-graphs - can be understood order by order without problems regarding the divergence of said generating functionals in quantum field theory. In this paper we focus on the first of the two steps, i.e. the change of the Lagrangian density $\mathcal{L}_{\phi} \mapsto \mathcal{L}_{\rho}=\mathcal{L}_{\phi}(\phi(\rho))$ which is induced by a transformation $\phi \mapsto \rho$ and examination of the Feynman rules and correlation functions of the theory defined by $\mathcal{L}_{\rho}$.

This paper is a continuation of earlier work by Kreimer, Yeats and Velenich [16, 17]. In the remainder of this section and Section 2, we will set up notation and review the results of [17], which mainly consider a diffeomorphism of an underlying free theory. Especially, the diffeomorphism field $\rho$ contains vertices which cancel adjacent propagators and thereby change the topology of graphs they reside in. We discuss 
this effect in great detail and introduce the connected perspective (Section 2.4), an alternative set of Feynman rules which automatically incorporates those internal cancellations and disentangles evaluation of graphs from topology changes.

Many concepts developed in Section 2 can be readily applied to diffeomorphisms of an underlying interacting theory, which will be done in Section 3. Next, we proceed in Section 4 to a possible application. Namely, we show that a diffeomorphism can for offshell correlation functions - alter the type of interaction present in the theory. In Section 5 we note that the results of Section 2 for the free theory are not limited to the specific momentum dependece of the propagator used there. Finally, in Section 6 we present a possibility of including derivatives into the transformation $\phi \mapsto \rho$ such that the welcome combinatorial structure of the global diffeomorphism is conserved.

\subsection{Prerequisites}

A Lagrangian is a power series in the field variable $\phi$, hence replacing $\phi$ by $\phi(\rho)$ according to Eq. 1.2 amounts to an insertion of power series into each other. For formal power series, the coefficients of the concatenation are given by Faà di Bruno's formula [7]: If

then

$$
f(t)=\sum_{n=1}^{\infty} f_{n} t^{n} \quad \text { and } \quad g(t)=\sum_{n=0}^{\infty} g_{n} t^{n}
$$

$$
\left[t^{n}\right](f(g(t)))=\frac{1}{n !} \sum_{k=1}^{n} k ! f_{k} \cdot B_{n, k}\left(1 ! g_{1}, 2 ! g_{2} \ldots,(n+1-k) ! g_{n+1-k}\right) .
$$

Here, $\left[t^{n}\right]$ denotes extraction of the $n^{\text {th }}$ coefficient (i.e. $\left[t^{n}\right] f(t)=f_{n}$ ) and $B_{n, k}$ are the partial Bell polynomials, defined via

$$
\sum_{n=0}^{\infty} \sum_{k=0}^{n} B_{n, k}\left(x_{1}, x_{2}, \ldots\right) u^{k} \frac{t^{n}}{n !}=\exp \left(u \sum_{j=1}^{\infty} x_{j} \frac{t^{j}}{j !}\right) \text {. }
$$

Bell Polynomials count the possible partitions of $\{1, \ldots, n\}$ into $k$ nonempty disjoint subsets:

$$
B_{n, k}\left(x_{1}, x_{2}, x_{3}, \ldots\right)=\sum_{P} x_{\left|P_{1}\right|} \cdots x_{\left|P_{k}\right|}
$$

where

$$
P=\left\{\emptyset \neq P_{i} \subseteq\{1, \ldots, n\} \forall i, \quad P_{i} \cap P_{j}=\emptyset \forall i \neq j, \quad P_{1} \cup \ldots \cup P_{k}=\{1, \ldots, n\}\right\} .
$$

Inversion of a power series is given by Lagrange inversion [4], which again applies for formal power series regardless of convergence as functions [12]:

$$
\left(f^{-1}\right)_{n}=\frac{1}{n !} \sum_{k=1}^{n-1} \frac{1}{f_{1}^{n+k}} B_{n-1+k, k}\left(0,-2 ! f_{2},-3 ! f_{3}, \ldots\right), \quad\left(f^{-1}\right)_{1}=\frac{1}{f_{1}} .
$$

We note in passing that concatenation and inversion of formal power series can also be interpreted as coproduct and antipode of the Faà di Bruno Hopf algebra [6]. 
For fixed $a \in \mathbb{N}_{0}$ and $b \in \mathbb{N}$ the Fuss-Catalan numbers are defined as

$$
F_{m}(a, b)=\frac{b}{m a+b}\left(\begin{array}{c}
m a+b \\
m
\end{array}\right)=b \frac{(m a+b-1) !}{(m a+b-m) ! m !} .
$$

We will also use the Euler characteristic which relates the number of vertices $\left|V_{\Gamma}\right|$, internal edges $\left|E_{\Gamma}\right|$ and loops $|\Gamma|$ of a connected graph $\Gamma$ :

$$
\left|V_{\Gamma}\right|-\left|E_{\Gamma}\right|+|\Gamma|=1 \text {. }
$$

\section{Diffeomorphisms of a Free Field}

This section discusses the case where an originally free scalar theory, defined via (1.1), is transformed by a diffeomorphism (1.2). We develop the "connected perspective" as a systematic tool to compute amplitudes in the diffeomorphed theory. Many of the results of Section 2 are already contained in [17] but will be useful in the later sections.

\subsection{Feynman Rules of the Ordinary Perspective}

Definition 2.1 (Offshell variable $s_{p}$ ) For a 4-momentum $p$ in a theory with mass $m \geq 0$ and Lagrangian (1.1), the corresponding offshell variable is defined as

$$
s_{p}:=p^{2}-m^{2} \text {. }
$$

This generalizes to sums of numbered momenta in a slight abuse of notation, e.g. let $p_{2}, p_{5}$ be two 4-momenta, then $s_{2+5}:=s_{p_{2}+p_{5}} \equiv\left(p_{2}+p_{5}\right)^{2}-m^{2}$. If momenta are not numbered but instead identified by the edges they are flowing through, the offshell variable also uses this labeling, e.g. $s_{e}:=p_{e}^{2}-m^{2}$ where $e$ is some edge in a graph with momentum $p_{e}$ flowing through it.

Applying the diffeomorphism (1.2) to the free Lagrangian (1.1) and collecting the derivatives of the kinetic term by partial integration yields

$$
\mathcal{L}_{\rho}:=\mathcal{L}_{\phi}(\phi(\rho))=-\sum_{n=1}^{\infty} \frac{f_{n+1}}{n !} \rho^{n} \partial_{\mu} \partial^{\mu} \rho-m^{2} \sum_{n=2}^{\infty} \frac{c_{n-2}}{n !} \rho^{n} .
$$

The quantities $f_{n}, g_{n}$ appear as coupling constants induced by the diffeomorphism.

$$
\begin{aligned}
f_{n} & =B_{n-2,1}\left(2 ! a_{1}, 3 ! a_{2}, \ldots\right)+B_{n-2,2}\left(2 ! a_{1}, 3 ! a_{2}, \ldots\right), \\
c_{n-2} & =B_{n, 2}\left(1,2 ! a_{1}, \ldots\right), \\
g_{n} & :=n f_{n}-c_{n-2}=\frac{n(n-2) !}{2} \sum_{k=0}^{n-2} a_{n-k-2} a_{k}(n-k-2) k .
\end{aligned}
$$

From Eq. 2.1, one reads off the $n$-valent vertex Feynman rules in momentum space

$$
i v_{n}=i f_{n} \cdot\left(s_{1}+\ldots+s_{n}\right)+i g_{n} \cdot m^{2}, \quad n \geq 2 .
$$

We will subsequently call these vertices diffeomorphism vertices. The first ones together with their explicit Feynman rules are depicted in Fig. 1. 


$$
\begin{aligned}
& i v_{3}=\stackrel{b}{\varrho}=2 i a_{1}\left(s_{1}+s_{2}+s_{3}\right) \\
& i v_{4}=\gamma^{\prime}=4 i m^{2} a_{1}^{2}+i\left(6 a_{2}+4 a_{1}^{2}\right)\left(s_{1}+s_{2}+s_{3}+s_{4}\right)
\end{aligned}
$$

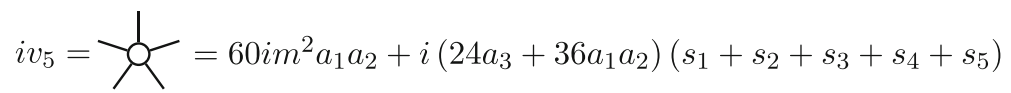

Fig. 1 Graphical representation of the diffeomorphism vertices $i v_{n}$ from Eq. 2.2. The numbered momenta correspond to edges adjacent to the vertex

In $D$ spacetime dimensions, the mass dimension of the fields is $[\phi]=\frac{D-2}{2}=[\rho]$, consequently

$$
\left[a_{j}\right]=-j \frac{D-2}{2}, \quad\left[v_{j}\right]=D-j \frac{D-2}{2} .
$$

\subsection{Tree Sums with One External Edge Offshell}

Since the diffeomorphism (1.2) is tangent to identity, the 2-point vertex is unaltered. The field $\rho$ has the same propagator (=non-amputated time ordered 2-point function) as $\phi$, with Definition 2.1

$$
\Gamma_{2, \text { free }}^{-1}=\langle\rho(p) \rho(-p)\rangle=\frac{i}{p^{2}-m^{2}}=\frac{i}{s_{p}} .
$$

Definition 2.2 (Tree sum $b_{n}$ ) The tree sums $b_{n}$ for $n \geq 3$ are defined as the sum of all trees with a total of $n$ external edges, where $n-1$ external edges are onshell (i.e. $s_{e}=0$ for these edges $e$ ) and additionally one external edge offshell. The propagator $\frac{i}{s_{e}}$ of this offshell edge $e$ is included in $b_{n}$. Further, $b_{2}:=1$.

Note that the index of $b_{n}$ is shifted by one in Definition 2.2 compared to [17, Section 3.1]. The construction of $b_{n}$ is illustrated in Example 2.3 and Fig. 2.

$$
b_{2}=b_{1}=1
$$

Fig. 2 The first tree sums $b_{n}$. Onshell edges are indicated with a perpendicular line. $b_{n}$ is drawn as a rectangle to distinguish it from diffeomorphism vertices $i v_{n}$ 
Example 2.3 (Tree sums $b_{n}$ ) An explicit calculation (see Example 2.11) using the vertices (2.2) yields

$$
\begin{aligned}
& b_{3}=\left.\frac{i}{s_{1+2}} \cdot 2 i a_{1}\left(s_{1}+s_{2}+s_{1+2}\right)\right|_{s_{1}=0=s_{2}}=-2 a_{1} \\
& b_{4}=-6 a_{2}+12 a_{1}^{2} .
\end{aligned}
$$

The tree sums $b_{n}$ computed in Example 2.3 are independent of masses and momenta. The following remarkable result was shown in [17, Theorem 3.5] explicitly using the propagator $i\left(p^{2}-m^{2}\right)^{-1}$ :

\section{Theorem 2.4}

$$
b_{n+2}=\sum_{k=1}^{n} \frac{(n+k) !}{n !} B_{n, k}\left(-1 ! a_{1},-2 ! a_{2}, \ldots,-n ! a_{n}\right) .
$$

Apart from their interpretation as Feynman amplitudes of tree sums, the $b_{n}$ also have an equally remarkable second interpretation:

Lemma 2.5 The tree sums $b_{n}$ are coefficients of $\rho(\phi)$, the inverse diffeomorphism of Eq. 1.2:

$$
\rho(x)=\sum_{n=1}^{\infty} \frac{b_{n+1}}{n !} \phi^{n}(x) .
$$

Proof Compute the coefficients of the inverse diffeomorphism using Lagrange inversion (1.6). Extracting coefficients from Eq. 1.4, one confirms

$$
B_{n+k, k}\left(0,-2 ! a_{1},-3 ! a_{2}, \ldots\right)=\frac{(n+k) !}{n !} B_{n, k}\left(-1 ! a_{1},-2 ! a_{2},-3 ! a_{3}, \ldots\right) .
$$

Definition 2.6 (Tree sum $A_{n}^{j}$ ) $A_{n}^{j}$ is the sum of all trees built from diffeomorphism vertices $i v_{k}$, with a total of $n$ external edges. At most $j$ of the $n$ external edges are offshell. External propagators are not included in $A_{n}^{j}$ and the sum is symmetrized over all ways to assign the offshell edges.

Note the difference regarding symmetry between Definitions 2.6 and 2.2: The tree sum $b_{n}$ has one distinguished offshell edge, the corresponding sum $A_{n}^{1}$ is the symmetric sum over all possibilities to let one of the $n$ external edges be offshell. Further, the external propagator $\frac{i}{s_{n}}$ is included in $b_{n}$ but not in $A_{n}^{1}$. Hence they are related by

$$
A_{n}^{1}=-i\left(s_{1}+s_{2}+\ldots+s_{n}\right) b_{n} .
$$

The requirement "at most $j$ offshell edges" in Definition 2.6 implies that the amplitudes $A_{n}^{j}$ have a recursive structure with regard to $j$ :

$$
\left.A_{n}^{j+1}\right|_{\text {only } j \text { edges offshell, symmetrized }}=A_{n}^{j} \text {. }
$$


This recursion does not imply that $A_{n}^{j+1}$ is necessarily different from $A_{n}^{j}$, see Example 2.18.

Lemma 2.7 If $A_{n}^{0}$ from Definition 2.6 is the connected tree level Feynman amplitude with $n>2$ external edges, all of which are onshell, then

$$
A_{n}^{0}=0 \text {. }
$$

Proof Set all $s_{j}=0$ in Eq. 2.5 to obtain the amplitude $A_{n}^{0}$ where none of the edges is offshell.

\subsection{Tree Sums as Vertices}

A tree, if it is not a single vertex, contains internal edges. Let $e$ be an internal edge, then the momentum $p_{e}$ flowing through it is a polynomial in the external momenta of the tree. This edge $e$ gives rise to a factor $\frac{i}{s_{e}}$ in the Feynman amplitude of the tree. Hence, if a tree contains $m$ internal edges, it a priori has a Feynman amplitude which is a rational function of the external offshell variables, of order $m$ in the denominator. The surprising result of Theorem 2.4 and Eq. 2.5 is that this is not the case for diffeomorphism Feynman rules: The tree sum $A_{n}^{1}$ is a polynomial, not a rational function, of the momenta adjacent to it. It thus has a Feynman amplitude as if it were a single vertex, there are no traces left of the internal propagators in the trees which have contributed to $A_{n}^{1}$.

Definition 2.8 (Metavertex) A metavertex is a symmetric sum of trees such that the total Feynman amplitude is a polynomial in the offshell variables Definition 2.1 of the external edges.

Such behaviour is very uncommon for Feynman amplitudes and gives rise to major confusion in the case of diffeomorphism Feynman rules: Evaluating the Feynman amplitude of a sum of trees in this case can produce the Feynman amplitude of a vertex, that is, an object which can be represented by a different Feynman graph. The diffeomorphism vertex Feynman rules (2.2) can in general alter the topology of the Feynman graph they are inserted in by cancelling edges.

\subsection{Ordinary and Connected Perspective}

To disentangle the topology-change from the usual evaluation of Feynman graphs, we will distinguish between two equivalent perspectives, the ordinary perspective and the connected perspective. These two perspectives apply not only to trees, but also to graphs including loops.

Definition 2.9 (1PI graph) A Feynman graph is 1-particle-irreducible (1PI) if it is 2-connected, that is, if it can not be decomposed into two disjoint parts by removing a single internal edge. 
Especially, a tree is not 1PI unless it is a single vertex. Feynman amplitudes of non-1PI graphs are products of the amplitudes of their 1PI components, therefore in ordinary perturbative QFT it is sufficient to compute the amplitudes of 1PI graphs. Eventually, the 1PI graphs are joined in all possible ways to produce the connected (but not necessarily 1PI) amplitudes, which are needed to compute scattering cross sections via the LSZ formalism [18].

The diffeomorphism vertex Feynman rules (2.2) are capable of changing the topology of the graph they reside in by cancelling internal edges. Especially, we saw in Section 2.3 that the sum of all trees with one external edge can be interpreted as a single vertex. This suggests that, after all cancellations are carried out, there arises a new set of graphs with vertex Feynman rules different from Eq. 2.2. We call these graphs the connected perspective of the corresponding amplitude.

Ordinary Perspective The ordinary perspective is the conventional way of working with Feynman graphs, known from qft textbooks: The Feynman rules of the diffeomorphed theory are Eqs. 2.2 and 2.4 as derived from the Lagrangian (2.1). The 1PI $n$-point-amplitude is the sum of all 1PI graphs with $n$ external edges. The connected amplitude is obtained by glueing 1PI graphs.

Connected perspective The connected perspective describes the sum of all connected graphs of the ordinary perspective contributing to a $n$-point-amplitude at a given loop order. In the connected perspective, all possible cancellations of internal edges have been carried out, giving rise to new Feynman rules for the vertices (called metavertices Definition 2.8) and certain restrictions on the construction of Feynman graphs, summarized in Theorem 2.17. The notion of 1PI amplitudes does not exist in the connected perspective. A single graph of the connected perspective already represents a sum of (usually non-1PI) connected graphs of the ordinary perspective.

Critically, the amplitudes obtained in the connected perspective coincide with the ones of the sum of all connected graphs of the ordinary perspective. The distinction is not about obtaining a different amplitude, but about interpreting the same amplitude in different ways. A metavertex of the connected perspective represents a sum of trees in the sum of graphs of the ordinary perspective. If the vertices of the ordinary perspective are momentum-independent, both perspectives coincide, i.e. these ordinary vertices become the only metavertices.

In the following, we shall develop the Feynman rules of the connected perspective.

Lemma 2.10 In the connected perspective of a free field diffeomorphism,

(1) A metavertex vanishes if all adjacent edges are onshell.

(2) A treelevel amplitude with precisely one edge offshell is given by a single metavertex

$$
V_{n}:=A_{n}^{1}=-i b_{n}\left(s_{1}+\ldots+s_{n}\right) .
$$

Proof (1) is Lemma 2.7, translated to the language of metavertices Definition 2.8. (2) is Eq. 2.5 as discussed in Section 2.3. We defined $V_{n}:=A_{n}^{1}$ to stress the importanc of just $A_{n}^{1}$ as a vertex. 
Note that the behaviour of the metavertex $V_{n}$ from Eq. 2.6 with respect to cancellation is more transparent than that of the ordinary vertex $v_{n}$ from Eq. 2.2: $V_{n}$ is a sum of terms, each of which cancels precisely one of the adjacent propagators. If that propagator is an external edge $e$ of the overall amplitude, there arises a factor $s_{e}$ which is nonzero only if the edge $e$ is offshell. Hence, cancelling an external edge requires that $e$ be offshell in order to give a non-vanishing result. On the contrary, $v_{n}$ includes additionally a constant summand $\propto m^{2}$ which does not cancel anything. $v_{n}$ does not vanish onshell.

Example 2.11 (Treelevel 4-point, one offshell edge) Ordinary perspective: With vertex Feynman rules (2.2), there are two different topologies contributing to the connected 4-point-amplitude, see the last row of Fig. 2: The 4-valent vertex (the external edges are numbered $1,2,3,4$ )

$$
i v_{4}=4 i m^{2} a_{1}^{2}+i\left(6 a_{2}+4 a_{1}^{2}\right)\left(s_{1}+s_{2}+s_{3}+s_{4}\right)
$$

and two 3-valent vertices glued together, which can appear in three different orientations,

$$
\begin{aligned}
\sum i v_{3} \frac{i}{s_{e}} i v_{3} & =2 i a_{1}\left(s_{1}+s_{2}+s_{1+2}\right) \frac{i}{s_{1+2}} 2 i a_{1}\left(s_{1+2}+s_{3}+s_{4}\right)+2 \text { other orientations } \\
& =-4 i a_{1}^{2}\left(s_{1+2}+s_{1+3}+s_{1+4}\right)-12 i a_{1}^{2}\left(s_{1}+s_{2}+s_{3}+s_{4}\right)+\mathcal{O}\left(s_{i} s_{j}\right) \\
& =-4 i a_{1}^{2} m^{2}-16 i a_{1}^{2}\left(s_{1}+s_{2}+s_{3}+s_{4}\right)+\mathcal{O}\left(s_{i} s_{j}\right)
\end{aligned}
$$

where we have used momentum conservation $s_{1+2}+s_{1+3}+s_{1+4}=s_{1}+s_{2}+s_{3}+$ $s_{4}+m^{2}$. In total,

$$
i v_{4}+\sum i v_{3} \frac{i}{s_{e}} i v_{3}=i\left(6 a_{2}-12 a_{1}^{2}\right)\left(s_{1}+s_{2}+s_{3}+s_{4}\right)+\mathcal{O}\left(s_{i} s_{j}\right) .
$$

The term $\mathcal{O}\left(s_{i} s_{j}\right)$ vanishes if only one external edge is offshell, i.e. if only one $s_{j} \neq$ 0 .

Connected perspective: By Lemma 2.10, a treelevel amplitude with one offshell edge is given by a single vertex with amplitude (2.6),

$$
V_{4}=-i b_{4}\left(s_{1}+s_{2}+s_{3}+s_{4}\right)=i\left(6 a_{2}-12 a_{1}^{2}\right)\left(s_{1}+s_{2}+s_{3}+s_{4}\right) .
$$

As advertised, the results agree. This is no surprise since the $b_{n}$ used in $V_{n}=A_{n}^{1}$ are literally the same tree sums which appear in the ordinary perspective. The point is that in the ordinary perspective, a number of graphs have to be summed where delicate cancellations take place whereas in the connected perspective the result can be written down right away without any further cancellations.

\subsection{Connected Tree Sums with Multiple Edges Offshell}

From Lemma 2.10 we know that there is a metavertex $V_{n}$ in the connected perspective. In this section we show that it is actually the only metavertex for a diffeomorphism of a free field. 
The mechanism to compute connected amplitudes with more than one external edge offshell was described in [17, Section 4.2.1], we re-derive it in this section to motivate the Feynman rules of the connected perspective. To this end, consider two metavertices $A_{m}^{1}, A_{n-m+2}^{1}$ which are joined by an internal edge $e$. The resulting amplitude has $n$ external edges and a priori contains three different types of summands, depending on whether $e$ is the cancelled edge of the adjacent metavertices.

(1) $A_{m}^{1}$ and $A_{n-m+2}^{1}$ both cancel one of their respective external edges and the internal edge $e$ is left uncancelled. Call the resulting sum of graphs $T_{m, n-m+2}$.

(2) $A_{m}^{1}$ cancels the internal edge and $A_{n-m+2}^{1}$ one of its $(n-m+1)$ external edges or vice versa.

(3) $A_{m}^{1}$ and $A_{n-m+2}^{1}$ both cancel the internal edge $e$, no external edge is cancelled.

In each of the cases, a symmetric sum over all permutations of external edges is understood.

Lemma 2.12 In the connected perspective, a metavertex $V_{j}$ must not cancel an internal edge.

Proof Cases (2) and (3) above. Case (3) corresponds - in the ordinary perspective - to the sum of all trees where no external edge is offshell. This sum is zero by Lemma 2.7. Case (2) represents the sum of all trees where precisely one external edge is offshell. This sum is $A_{n}^{1}=V_{n}$, a metavertex itself. Therefore, by allowing an internal edge between two metavertices to be cancelled by one of them, one overcounts the part of the amplitude which has one less external edge offshell. To avoid this, one must demand that no internal edge is cancelled by any $V_{j}$.

Definition 2.13 (Internal and external vertices) Let $\Gamma$ be a graph. A vertex $v \in \Gamma$ is called external if it is adjacent to at least one of the external edges of $\Gamma$. Otherwise, $v$ is called internal.

Lemmas 2.10 and 2.12 together imply that graphs in the connected perspective do not contain internal metavertices $V_{j}$.

Lemma 2.14 In the connected perspective, a tree with $j$ metavertices Definition 2.8 of type $V_{k}$ has at least $j$ external edges offshell.

Proof By Lemma 2.10, each metavertex cancels at least one of its adjacent edges. By Lemma 2.12, no internal edge must be cancelled. Therefore, a tree with $j$ metavertices cancels at least $j$ external edges, i.e. it involves factors $s_{e}$ for those edges $e$. If one of the cancelled external edges is onshell, $s_{e}=0$, then the amplitude vanishes. Hence, at least $j$ external edges are offshell. 
Lemma 2.15 In the connected perspective of a diffeomorphism of a free field, $V_{n}$ from Eq. 2.6 is the only type of metavertex (Definition 2.8).

Proof By Lemma 2.10 there is no momentum-independent metavertex. To be shown: There is no metavertex of higher than first order in its external offshell variables.

This is a consequence of mass dimension. Using Eq. 2.3, $\left[b_{j}\right]=\left[a_{j-2}\right]=$ $D-2-j \frac{D-2}{2}$. Since $\left[s_{j}\right]=+2,\left[V_{n}\right]=\left[s_{j}\right]+\left[b_{n}\right]=D-n \frac{D-2}{2}$. Assume there is a metavertex at least quadratic in the external offshell variables, $T_{n}:=t_{n} \sum_{i, k} s_{i} s_{j}$. In the ordinary perspective, it is built from trees with $n$ external edges just like $V_{n}$. But the mass dimension of the diffeomorphism vertices Eq. 2.3 is well defined, i.e. fixing the number of external edges of a tree fixes its overall mass dimension. Therefore, $\left[T_{n}\right]=\left[V_{n}\right]$ and consequently $\left[t_{n}\right]=\left[b_{n}\right]-2$. On the other hand, to be a metavertex Definition 2.8, $T_{n}$ must not contain momenta in the denominator. The only remaining possibility is that $t_{n}$ be of higher order in $a_{j}$ than $b_{n}$. But order in $a_{j}$ implies valence of the diffeomorphism vertices Eq. 2.2, and it is not possible to replace a vertex in a tree with another vertex of higher valence without changing the number of external edges. Consequently, the number of external edges fixes the order in $a_{j}$ and therefore the mass dimension of any momentum-independent factor that can arise in the metavertex Feynman rules.

Note that this does not mean that $A_{k}^{j}$ (Definition 2.6), tree sums with more than one external offshell edge, vanish. They do not vanish, but they can not be interpreted as a single vertex in the connected perspective because they involve internal propagators.

Lemma 2.16 In the connected perspective, $A_{n}^{2}$, the treelevel $n$-point amplitude with at most two external edges offshell, is given by two contributions:

(1) A single metavertex $V_{n}$

(2) A sum over all ways to choose $i+j=n+2$ and connect two metavertices $V_{i}, V_{j}$ by an uncancelled internal edge.

Proof The contribution of the single metavertex represents the amplitude with only one external edge offshell. By Lemma 2.14, there is no contribution from trees with more than two metavertices. By Lemma 2.15, there is no contribution from any other single vertex than $V_{n}$.

The sum (2) represents all trees which can be built from two metavertices. In the ordinary perspective, each $V_{j}$ corresponds to a sum over all $j$-valent trees, therefore, the sum (2) equals the sum over all trees where exactly one internal edge is left uncancelled. Following the proof of Lemma 2.15, such trees are the only ones wich can produce an overall factor quadratic in external offshell variables.

Lemma 2.16 immediately generalizes to a higher number of external offshell edges. The Euler characteristic Eq. 1.8 ensures that a tree with $j$ metavertices has $j-1$ internal edges. By Lemmas 2.10, 2.12 and 2.15 each metavertex cancels precisely one external edge which therefore needs to be offshell. Consequently, a tree 
sum with $j$ external offshell edges is - in the connected perspective - the sum of all trees with up to $j$ metavertices $V_{k}$ (with not necessarily the same $k$ ).

\subsection{Feynman Rules of the Connected Perspective}

This theorem presents all the Feynman rules of the connected perspective of Section 2.4. Loop-level amplitudes have not yet been discussed but they are included for later reference.

Theorem 2.17 (Feynman rules of the connected perspective of a free field diffeomorphism)

Assuming vanishing of tadpoles, the n-point connected amplitude is obtained by summing over all connected graphs $\Gamma$ such that

(1) Each internal edge e contributes a propagator factor $\frac{i}{s_{e}}$.

(2) $\Gamma$ is built from $(k>2)$-valent metavertices with amplitude $V_{k}=-i b_{k}\left(s_{1}+\right.$ $\left.s_{2}+\ldots+s_{k}\right)$. Keeping a summand $s_{e}$ in this amplitude amounts to cancelling the adjacent edge $e$.

(3) The metavertices do not cancel internal edges of $\Gamma$.

(4) There are no internal (Definition 2.13) metavertices.

Proof For treelevel, this is a combination of Eq. 2.4 and Lemmas 2.10, 2.12, 2.15 and 2.16. For loop graphs, the same rules hold as long as tadpole graphs vanish as will be discussed in Section 2.7.

Point (4) is redundant, but it is kept for clarity. There are no two-valent metavertices, hence a tree with $n$ external edges has at most $n-2$ metavertices. Consequently, at most $n-2$ of its external edges can be cancelled. For the treelevel amplitudes of Definition 2.6 this implies

$$
A_{n}^{n}=A_{n}^{n-2}
$$

Example 2.18 (Treelevel 4-point, all edges offshell) Computing explicitly in the ordinary perspective as outlined in Example 2.11, the treelevel amplitude with four external edges is

$$
A_{4}^{4}=-i b_{4}\left(s_{1}+s_{2}+s_{3}+s_{4}\right)+\left(-i b_{3}\right)\left(\left(s_{1}+s_{2}\right) \frac{i}{s_{1+2}}\left(s_{3}+s_{4}\right)+2 \text { more }\right)\left(-i b_{3}\right) .
$$

This is precisely the result expected from the Feynman rules Theorem 2.17. We depict $V_{n}$ like $b_{n}$ (compare Fig. 2):

$$
A_{4}^{4}=\sum_{4 \text { Perm. }}
$$

There is a 4-valent metavertex $V_{4}=-i b_{4}\left(s_{1}+s_{2}+s_{3}+s_{4}\right)$ and two 3-valent metavertices $V_{3}=-i b_{3}\left(s_{1}+s_{2}+s_{3}\right)$ glued toghether by an internal propagator which remains uncancelled. Hence, each of these vertices contributes only two (and not all three) summands $s_{j}$ corresponding to the two external edges adjacent to it. 
Finally, there is no contribution of third order in the external momenta, hence $A_{4}^{4}=$ $A_{4}^{2}$ as claimed in Eq. 2.7.

Example 2.19 (Treelevel 6-point, all edges offshell) The amplitude with six external edges is the first case where Lemma 2.12 resp. Theorem 2.17 (4) restricts topology in the connected perspective. In the ordinary perspective, it would be possible to have a tree with four 3-valent vertices, three of them connected to a central internal (Definition 2.13) one. In the connected perspective no such tree contributes, the central metavertex is zero because it must not cancel one of its adjacent edges.

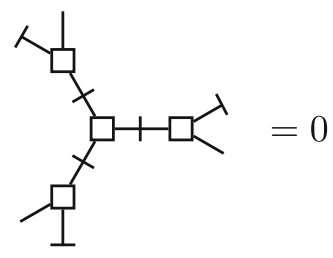

The contribution of this graph is already contained in trees consisting of three metavertices.

There are six possible topologies of trees without internal vertices, they give rise to the amplitude

$$
\begin{aligned}
A_{6}^{6}=A_{6}^{4}=-i b_{6} & \left(s_{1}+s_{2}+s_{3}+s_{4}+s_{5}+s_{6}\right) \\
& +\left(-i b_{3}\right)\left(s_{1}+s_{2}\right) \frac{i}{s_{1+2}}\left(-i b_{5}\right)\left(s_{3}+s_{4}+s_{5}+s_{6}\right)+14 \text { more permutations } \\
& +\left(-i b_{4}\right)\left(s_{1}+s_{2}+s_{3}\right) \frac{i}{s_{1+2+3}}\left(-i b_{4}\right)\left(s_{4}+s_{5}+s_{6}\right)+19 \text { more permutations } \\
& +\left(-i b_{3}\right)\left(s_{1}+s_{2}\right) \frac{i}{s_{1+2}}\left(-i b_{3}\right) s_{3} \frac{i}{s_{1+2+3}}\left(-i b_{4}\right)\left(s_{4}+s_{5}+s_{6}\right)+59 \text { more } \\
& +\left(-i b_{3}\right)\left(s_{1}+s_{2}\right) \frac{i}{s_{1+2}}\left(-i b_{4}\right)\left(s_{3}+s_{4}\right) \frac{i}{s_{5+6}}\left(-i b_{3}\right)\left(s_{5}+s_{6}\right)+44 \text { more } \\
& +\left(-i b_{3}\right)\left(s_{1}+s_{2}\right) \frac{i}{s_{1+2}}\left(-i b_{3}\right) s_{3} \frac{i}{s_{1+2+3}}\left(-i b_{3}\right) s_{4} \frac{i}{s_{5+6}}\left(-i b_{3}\right)\left(s_{5}+s_{6}\right)+89 \text { more. }
\end{aligned}
$$

\subsection{Loop Amplitudes and Tadpoles}

The Feynman rules of the connected perspective (Theorem 2.17) are suitable for the construction of graphs including loops up to one peculiarity regarding the cancellation of self-loops.

Definition 2.20 (Tadpole) A Tadpole Feynman integral is an integral over a loop momentum which is independent of the external momenta of the Feynman amplitude.

Depending on the regularization scheme, a tadpole integral evaluates to a (possibly infinite) constant. In terms of Feynman graphs, a tadpole graph is a Feynman graph where there is at least one subgraph which is connected to the rest of the graph via a single vertex and has no external edges itself. But this graphical identification assumes that no internal edges of the graph are cancelled, i.e. it works only in the connected perspective of Section 2.4. In the ordinary perspective of Section 2.4 on 
the other hand, there can be a "hidden" (i.e. not obvious in the graphical representation) cancellation of internal edges which turns a seemingly non-tadpole graph into a tadpole-type graph, see Fig. 3.

The connected perspective rests on the finding that none of the internal edges must be cancelled by metavertices (Lemma 2.12). This is because in a tree, such a cancellation between two vertices produces an amplitude which already is present in a metavertex replacing both vertices, i.e. the cancellation would introduce doublecounting of graphs. This argument no longer holds in the case of loop amplitudes as soon as there are tadpoles. A self-loop involves only one single vertex, hence there is no bigger metavertex which could possibly include this cancelled contribution. Consequently, the metavertices are allowed to cancel self-loops. But such cancellation gives rise to a (violently divergent) tadpole integral which is not visible in the graphical representation. If a self-loop connected to a metavertex $V_{n}$ is cancelled, one obtains a new type of $(n-2)$-valent, momentum independent metavertex, see Exampled 2.21. That is, if tadpole graphs do not vanish then there is (at least) one additional type of metavertex in the connected perspective. This metavertex represents a constant infinite contribution to the $S$-matrix.

In a renormalizable theory, such constant terms are removed in the renormalization process which is especially straightforward in kinematic renormalization schemes. In our case, there is no such automatic mechanism, so there are two choices: Either one accepts that infinite constants be added to the $S$-matrix or one requires the vanishing of tadpoles as an axiom at this point. We choose the latter and leave out all tadpole graphs in the following. This procedure is, regardless of renormalizability of the theory, at least self-consistent since tadpoles are a Hopf-ideal in the Hopf-algebra of Feynman graphs [2]. Technically, leaving out tadpoles can be understood as a type of renormalization: Tadpole amplitudes can be cancelled by a suitable redefinition of vertices. However, it is not a classical renormalization in the sense that this procedure is not sufficient to render all amplitudes finite even for offshell momenta.
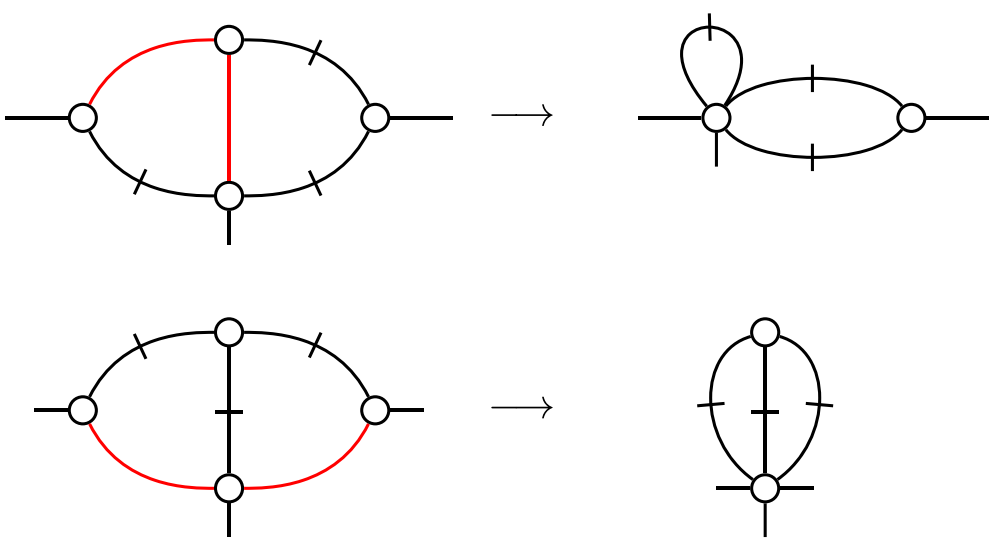

Fig. 3 Two different ways to obtain tadpoles from non-tadpole graphs by cancellation of internal edges in the ordinary perspective. Cancelled edges are red, uncancelled ones have a perpendicular line 
Example 2.21 (Tadpole with 5-valent metavertex) Consider the graph $\Gamma$ which is a 1-loop correction to the connected 3-point amplitude.

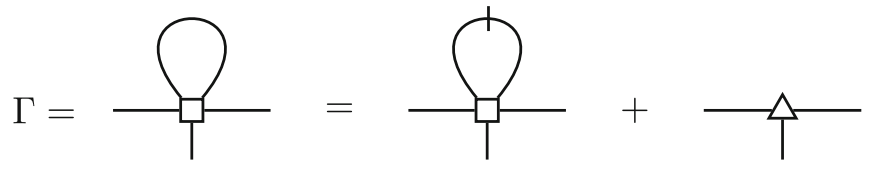

The 5-valent metavertex $V_{5}=-i b_{5}\left(s_{1}+s_{2}+s_{3}+s_{4}+s_{5}\right)$ cancels either one of the three external edges or the self-loop. The Feynman amplitude of this graph, including the symmetry factor, can be decomposed into two summands,

$$
\begin{aligned}
\Gamma & =\frac{1}{2} \int \mathrm{d}^{D} k\left(-i b_{5}\right)\left(s_{1}+s_{2}+s_{3}+s_{k}+s_{k}\right) \frac{i}{s_{k}} \\
& =\frac{1}{2}\left(-i b_{5}\right)\left(s_{1}+s_{2}+s_{3}\right) \int \mathrm{d}^{D} k \frac{i}{s_{k}}+b_{5} \int \mathrm{d}^{D} k 1 .
\end{aligned}
$$

These two terms are indicated graphically as well. The first one represents the case where the metavertex cancels one of the external edges, the second one effectively is a 3 -valent metavertex. This is however a new type of metavertex which does not cancel any edges at all. Instead, the Feynman rule for this new vertex is an infinite constant. Renormalization then amounts to redefinition of the 3-valent metavertex in order to eliminate this constant. Leaving out tadpole graphs in the connected perspective guarantees that no such metavertices arise.

Theorem 2.17 implies that a connected amplitude with $j$ external offshell edges contains at most $j$ metavertices $V_{k}$. On the other hand, by Definition 2.20 it contains at least two such metavertices in order to not be a tadpole. Hence, the amplitude vanishes if less than two external edges are offshell. Especially, the $S$-matrix [11] is unaffected by the diffeomorphism and graphs with two external edges have the topology of $l$-loop multiedges [17, lemma 4.8] .

\subsection{Alternative Proof of the Tree Sum Feynman Amplitude}

A first application of the Feynman rules in the connected perspective is an alternative derivation of Theorem 2.4. To this end, note that the Feynman rules Theorem 2.17 do not use the explicit expression for $b_{n}$ in terms of $a_{j}$, they merely use $b_{n}$ as tree sums following Definition 2.2. First, we show that Lemma 2.5 holds on graph-theoretical grounds independent of Theorem 2.4:

Lemma 2.22 The coefficients $b_{n}$ of the inverse

$$
\rho(x)=\sum_{n=1}^{\infty} \frac{b_{n+1}}{n !} \phi^{n}(x)
$$

of the diffeomorphism (1.2) are the Feynman amplitudes of amputated metavertices $V_{n}$ (i.e. they coincide with the amplitudes of tree sums $b_{n}$ as defined in Definition 2.2). 
Proof Consider the non-amputated time-ordered 2-point correlation function of $\rho$. By Theorem 2.17 and vanishing of tadpoles, it is supported on $l$-loop multiedge graphs $M^{(l)}$ where the two vertices are metavertices $V_{l+2}$ (we denote by $M^{(l)}$ the Feynman amplitude of the amputated $l$-loop multiedge). The single offshell edge of said vertices carries momentum $p$ and the graph $M^{(l)}$ has a symmetry factor $\frac{1}{(l+1) !}$. Including the two external propagators, one has

$$
\langle\rho(-p) \rho(p)\rangle=\langle\phi(-p) \phi(p)\rangle+\frac{i}{s_{p}} \sum_{l=1}^{\infty}\left(V_{l+2}\right)^{2} \cdot \frac{1}{(l+1) !} M^{(l)}(p) \frac{i}{s_{p}} .
$$

On the other hand, using Eq. 2.8 in position space (where we take $b_{n}$ to be the unknown coefficients of the inverse) the same function is

$$
\langle T \rho(x) \rho(y)\rangle=\sum_{j=1}^{\infty} \sum_{k=1}^{\infty} \frac{b_{j+1} b_{k+1}}{j ! k !}\left\langle T \phi^{j}(x) \phi^{k}(y)\right\rangle .
$$

The right hand side are $(j+k)$-point correlation functions of the field $\phi$ which are computed via Wick's theorem, i.e. all factors $\phi$ of the field have to be connected in pairs which eliminates all summands were $j+k$ is odd. If $j+k$ is even, any term where two fields at the same spacetime point are connected correspond - after Fourier transformation - to a tadpole integral which is assumed to vanish. Hence all non-vanishing pairs have to be of the form $\phi(x) \phi(y)$ which implies $j=k$,

$$
\langle T \rho(x) \rho(y)\rangle=\left\langle T \phi(x) \phi(y)+\sum_{k=2}^{\infty} \frac{\left(b_{k+1}\right)^{2}}{(k !)^{2}}\left\langle T \phi^{k}(x) \phi^{k}(y)\right\rangle .\right.
$$

There are precisely $k$ ! equivalent ways of forming pairs which cancels one factor in the denominator. The resulting sum equals after Fourier transformation the sum over all $(k-1)$-loop multiedges, weighted with their correct symmetry factor $\frac{1}{k !}$,

$$
\langle\rho(p) \rho(-p)\rangle=\langle\phi(-p) \phi(p)\rangle+\sum_{k=2}^{\infty} \frac{\left(b_{k+1}\right)^{2}}{k !} M^{(k-1)}(p) .
$$

Comparing coefficients in Eqs .2.9 and 2.10 yields the claimed equality

$$
\left.V_{l+2}\right|_{s_{1}=s_{p} \neq 0}=-i s_{p} b_{l+2} \text {. }
$$

This of course coincides with Eq. 2.6, but the meaning is completely different: Eqs. 2.5 and 2.6 are a definition of $V_{n}$ in terms of tree sums $b_{n}$, whereas here we showed that the metavertex $V_{n}$ necessarily coincides with the inverse diffeomorphism coefficients.

The proof implicitly relies on the existence of the connected perspective, i.e. the fact that tree sums with one external edge offshell evaluate to some constant. But then it assures that this constant can only possibly be the inverse diffeomorphism coefficient. 
Lemma 2.23 If $b_{n}$ is the Feynman amplitude of the tree sum Definition 2.2 then

$$
b_{n+2}=\sum_{k=1}^{n} \frac{(n+k) !}{n !} B_{n, k}\left(-1 ! a_{1},-2 ! a_{2}, \ldots,-n ! a_{n}\right) \text {. }
$$

Proof By Lemma 2.22, $b_{n}$ coincides with the corresponding series coefficient of the inverse diffeomorphism. On the other hand, the coefficient of the inverse diffeomorphism has the claimed formula by Lagrange inversion (1.6). Hence, that same expression also represents the amplitude of the tree sum $b_{n+2}$.

Apart from suggesting that the derived Feynman rules of the connected perspective are self-consistent, Lemma 2.23 is in a particular sense a generalization of Theorem 2.4 because it does not use the explicit form $i\left(p^{2}-m^{2}\right)^{-1}$ of the propagator (we mention this form in Theorem 2.17, but all that is used is actually the fact that diffeomorphism vertices cancel adjacent propagators). Therefore, Lemma 2.23 holds even for more exotic propagators as long as the cancellation-properties of the diffeomorphism vertices (2.2) stay intact. We explore this in more detail in Section 5. On the other hand, Lemma 2.23 relies on the vanishing of tadpoles, while Theorem 2.4 does not.

\section{Diffeomorphism of an Interacting Field}

\subsection{Feynman Rules of the Ordinary Perspective}

In this section, the diffeomorphism (1.2) is applied to a $\phi^{t}$-type interacting field, i.e.

$$
\mathcal{L}_{\phi}=\frac{1}{2} \partial_{\mu} \phi(x) \partial^{\mu} \phi(x)-\frac{1}{2} m^{2} \phi^{2}(x)-\frac{\lambda_{t}}{t !} \phi^{t}(x) .
$$

The coupling constant $\lambda_{t}$ carries an index $t$ to better keep track of the type of interaction.

Since the first part of Eq. 3.1 coincides with Eq. 1.1, the field $\rho$ obtains the same vertices $i v_{n}$ as in the free case (see Eq. 2.2). They once more combine to the amplitudes $A_{n}^{j}$ from Definition 2.6.

Additionally, the interaction monomial in Eq. 3.1 gives rise to a second type of vertex with Feynman rule

$$
-i w_{n}^{(t)}=-i \frac{\lambda_{t}}{t !} n ! \underbrace{\sum_{j=0}^{n-t} \sum_{k=0}^{n-t-j} \cdots \sum_{l=0}^{n-t-j-k-\ldots}}_{t-1 \text { sums }} \underbrace{a_{j} a_{k} \cdots a_{l} a_{n-t-j-k-\ldots-l}}_{t \text { factors } a} .
$$

The vertex Feynman rule is by construction $n$ ! times the coefficient of $\rho^{n}$ of the power series $\frac{\lambda_{t}}{t !} \phi^{t}(\rho)$. Hence using Eq. 1.3, Bell polynomials allow for a condensed notation of this multisum:

$$
-i w_{n}^{(t)}=-i \lambda_{t} B_{n, t}\left(1 ! a_{0}, 2 ! a_{1}, 3 ! a_{2}, 4 ! a_{3}, \ldots\right), \quad w_{n}^{(t)}=0 \forall n<t .
$$


As the diffeomorphism (1.2) is tangent to identity, it reproduces the lowest order term, i.e. there is a vertex $-i w_{t}^{(t)}=-i \lambda_{t}$ and no vertex $-i w_{n}^{(t)}$ where $n<t$. As an illustration, the diffeomorphism vertices of underlying $\phi^{3}$ and $\phi^{4}$-theory are shown in Fig 4.

Analogous to Eq. 2.1, the vertices $w_{n}^{(t)}$ from Eq. 3.5 can be interpreted as coupling constants, namely the Lagrangian of $\rho$ reads

$$
\mathcal{L}_{\rho}=-\sum_{n=1}^{\infty} \frac{f_{n+1}}{n !} \rho^{n}(x) \partial_{\mu} \partial^{\mu} \rho(x)+m^{2} \sum_{n=2}^{\infty} \frac{c_{n-2}}{n !} \rho^{n}(x)-\sum_{n=t}^{\infty} \frac{w_{n}^{(t)}}{n !} \rho^{n}(x)
$$

The construction works equivalently well in the case of multiple interaction terms in the original Lagrangian,

$$
\mathcal{L}_{\phi}=-\frac{1}{2} \phi(x) \partial_{\mu} \partial^{\mu} \phi(x)-\frac{1}{2} m^{2} \phi^{2}(x)-\sum_{t=3}^{\infty} \frac{\lambda_{t}}{t !} \phi^{t}(x)
$$

Each interaction monomial $\phi^{t}$ gives rise to a new family of interaction vertices (3.2). The total contribution to the $n$-valent interaction vertex is the sum of all $-i w_{n}^{(t)}$ where $t \leq n$,

$$
-i w_{n}:=-i \sum_{t=3}^{n} w_{n}^{(t)}=-i \sum_{t=3}^{n} \lambda_{t} B_{n, t}\left(1 !, 2 ! a_{1}, 3 ! a_{2}, \ldots\right)
$$

Together with the diffeomorphism vertex $i v_{n}$ from Eq. 2.2, the most general form of a $n$-valent vertex, which can arise through global diffeomorphism of a scalar

$$
\begin{aligned}
& \text { underlying } \phi^{3} \text { theory }(t=3) \\
& \text { underlying } \phi^{4} \text { theory }(t=4) \\
& \downarrow-i w_{3}^{(3)}=-i \lambda_{3} \\
& -i w_{3}^{(4)}=0 \\
& \gamma \quad-i w_{4}^{(3)}=-12 i \lambda_{3} a_{1} \\
& -i w_{4}^{(4)}=-i \lambda_{4} \\
& \mathcal{1}-i w_{5}^{(3)}=-60 i \lambda_{3}\left(a_{2}+a_{1}^{2}\right) \\
& -i w_{5}^{(4)}=-20 i \lambda_{4} a_{1} \\
& -i w_{6}^{(3)}=-120 i \lambda_{3}\left(3 a_{3}+6 a_{1} a_{2}+a_{1}^{3}\right) \quad-i w_{6}^{(4)}=-60 i \lambda_{4}\left(2 a_{2}+3 a_{1}^{2}\right)
\end{aligned}
$$

Fig. 4 Diffeomorphism-interaction vertices according to Eq. 3.5. They are drawn black to distinguish them from "pure" diffeomorphism vertices Fig. 1 
theory (3.4), is

$$
\begin{aligned}
i v_{n}-i w_{n}=i( & \left.B_{n-2,1}\left(2 ! a_{1}, 3 ! a_{2}, \ldots\right)+B_{n-2,2}\left(2 ! a_{1}, 3 ! a_{2}, \ldots\right)\right)\left(x_{1}+\ldots+x_{n}\right) \\
& +i m^{2}\left(n B_{n-2,1}\left(2 ! a_{1}, \ldots\right)+n B_{n-2,2}\left(2 ! a_{1}, \ldots\right)-B_{n, 2}\left(1 ! a_{0}, 2 ! a_{1}, \ldots\right)\right) \\
& \quad-i \sum_{t=3}^{n} \lambda_{t} B_{n, t}\left(1 !, 2 ! a_{1}, 3 ! a_{2}, \ldots\right)
\end{aligned}
$$

Note that - althought there are two possibly infinite sets of free parameters $\left\{\lambda_{t}\right\}_{t \geq 3}$ and $\left\{a_{j}\right\}_{j \geq 1}$ - the momentum dependence of this vertex is very restricted. This fact is a consequence of globality of the diffeomorphism: The diffeomorphism does not introduce additional derivatives and the underlying Lagrangian (3.4) only contains up to second derivatives, so no global diffeomorphism can produce a vertex with Feynman rule of higher than second order in momenta. Compare Section 6.

\subsection{Cancellation of Higher Interaction Vertices}

As soon as interaction is present in the underlying Lagrangian, by Eq. 3.5 an infinite set of new interaction vertices is generated by the diffeomorphism. Consider the sum of all tree graphs with $n$ external edges instead of only the vertex $-i w_{n}^{(t)}$, then almost all contributions cancel if the external edges are onshell. The only remaining terms arise from trees built of $t$-valent vertices $-i \lambda_{t}$. For underlying $\phi^{4}$-theory, this statement is [17, theorem 5.1]. Note also in [16] this cancellation is discussed based on properties of the $S$-matrix, here we instead use explicit identities for the Feynman rules to prove it.

Definition 3.1 (Tree sum $W_{n}^{(t)}$ ) $W_{n}^{(t)}$ is defined to be the sum of all trees proportional to $\lambda_{s}$ in the ordinary perspective of a diffeomorphism of $\phi^{t}$-theory which have $n$ external edges, all of which are onshell.

$W_{n}^{(t)}$ is one possible generalization of $A_{n}^{0}$ from Definition 2.6 and Lemma 2.7 to underlying interacting fields. Note that generally a $n$-valent tree sum also involves summands of higher than first order in $\lambda_{t}$, they are not included into $W_{n}^{(t)}$, see Eq. 3.10.

Example 3.2 (Tree sum $W_{4}^{(3)}$ ) Consider the tree sum with $n=4$ external edges in the diffeomorphism of $\phi^{3}$ theory, $t=3$. Using Definition 3.1, we restrict to terms of first order in $\lambda_{3}$, these are trees which contain precisely one vertex of type $-i w_{j}^{(3)}$ (all other vertices are of diffeomorphism type $i v_{k}$ ).

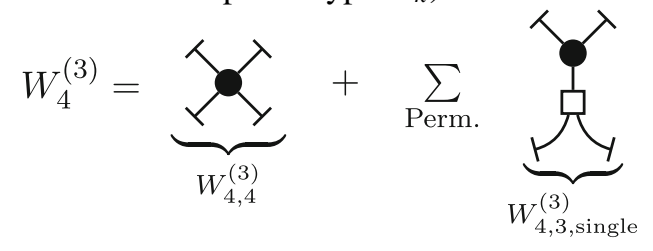


There are two contributions to $W_{4}^{(3)}$. The first, $W_{4,4}^{(3)}$, is the 4-valent interaction vertex

$$
W_{4,4}^{(3)}=-i w_{4}^{(3)}=-12 i \lambda_{3} a_{1} .
$$

The second contribution to $W_{4}^{(3)}$ is a sum over trees consisting of one interaction vertex and a tree sum Definition 2.2 (which happens to be $b_{3}$, just one vertex $i v_{3}$, here, see Example 2.3). There are six different permutations of the external edges, one of them is

$$
W_{4,3, \text { single }}^{(3)}=-i \lambda_{3} \cdot\left(-2 a_{1}\right)=2 i \lambda_{3} a_{1} .
$$

This does not depend on external momenta, therefore summing the six permutations produces

$$
W_{4,3}^{(3)}=6 \cdot W_{4,3, \text { single }}^{(3)}=12 i \lambda_{3} a_{1} .
$$

Hence, the total amplitude proportional to $\lambda_{3}$ is

$$
W_{4}^{(3)}=W_{4,4}^{(3)}+W_{4,3}^{(3)}=0 .
$$

Now let $t \geq 3$ be arbitrary but fixed. We will show the analogue of Lemma 2.7 for the interacting case, namely that all $W_{n}^{(t)}$ for $n>s$ are zero. This is based on two observations:

(1) Each interaction vertex $-i w_{n}^{(t)}$ is of order one in $\lambda_{t}$. For the evaluation of $W_{n}^{(t)}$, it is sufficient to restrict to trees where there is only one single interaction vertex and the remaining vertices are of pure diffeomorphism type $i v_{j}$ from Eq. 2.2.

(2) An interaction vertex $-i w_{n}^{(t)}$ does not cancel adjacent propagators, therefore such vertex can only be cancelled against a tree sum which also does not cancel propagators. Especially, one can require all $n$ external edges to be onshell.

Euler characteristic Eq. 1.8 ensures compatibility of these requirements: A tree $T$ with $\left|V_{T}\right|$ vertices has $\left|V_{T}\right|-1$ internal edges, and if one of the vertices is of $-i w_{j}^{(t)}$ type, the remaining $\left|V_{T}\right|-1$ vertices of $i v_{j}$ type precisely suffice to cancel all internal edges but none of the external ones. Summing over all possible trees and permutations of external edges, $W_{n}^{(t)}$ consists of summands where one vertex $-i w_{j}^{(t)}$ is connected to $j$ tree sums $b_{k_{1}}, \ldots, b_{k_{j}}$ such that $k_{1}+\ldots+k_{j}=n+j$. This is shown in Fig. 5 for $t=3$ (for other $t$, the sum ends at a $t$-valent interaction vertex on the right side).

The first term of $W_{n}^{(t)}$ only contains tree sums $b_{2} \equiv 1$, we call it $W_{n, n}^{(t)}$ (where the second $n$ indicates that a vertex $-i w_{n}^{(t)}$ is involved):

$$
W_{n, n}^{(t)}=-i w_{n}^{(t)} \underbrace{b_{2} b_{2} \cdots b_{2}}_{n \text { factors }} .
$$

The second contribution to $W_{n}^{(t)}$ contains one $b_{3}$ and the rest $b_{2}$, it is $W_{n,(n-1) \text {, single }}^{(t)}=$ $-i w_{n-1}^{(t)} b_{3} \cdot b_{2}^{n-2}$. The third term is made from a vertex $-i w_{n-2}^{(t)}$ and either one $b_{4}$ or two $b_{3}$ and the rest $b_{2}$. The last contribution to $W_{n}^{(t)}$, shown in the right in Fig. 5, has 


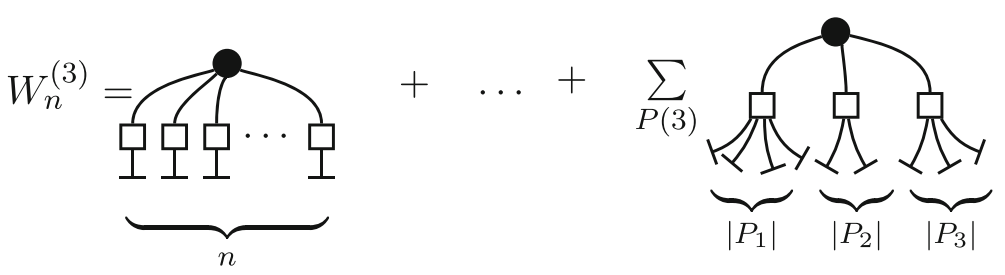

Fig. 5 Structure of the contributions to $W_{n}^{(3)}$ : The external edges are onshell, all terms consist of tree sums $b_{k}$ (for some $k$ ) and one interaction vertex $-i w_{j}^{(3)}$. The sum $P(k)$ runs over all ways of distributing $n$ external edges to $j$ tree sums $b_{\left|k_{i}\right|}$

one vertex $-i w_{t}^{(t)}$ and $t$ tree sums $b_{k_{1}}, b_{k_{2}}, b_{k_{t}}$ such that $k_{j} \geq 2, k_{1}+k_{2}+\ldots+k_{t}-t=$ $n$.

For any fixed valence $k$ of the interaction vertex $-i w_{k}^{(t)}$, there is a sum over the set $P(k)$ of all possible ways of assigning the $n$ external edges to the individual tree sums. This is the same partition as in the definition of Bell polynomials Eq. 1.5, hence for any fixed $k$

$$
W_{n, k}^{(t)}=-i w_{k}^{(t)} \sum_{P(k)} W_{n, k, \text { single }}^{(t)}=-i w_{k}^{(t)} \sum_{P(k)} \prod_{i=1}^{k} b_{k_{i}}=B_{n, k}\left(b_{2}, b_{3}, \ldots\right) .
$$

Finally, $W_{n}^{(t)}$ contains all of these terms for $k \in\{t, \ldots, n\}$. Inserting the Feynman rule (3.2) for $-i w_{n}^{(t)}$ yields

$$
W_{n}^{(t)}=-i \lambda_{t} \sum_{k=t}^{n} B_{k, t}\left(1,2 ! a_{1}, 3 ! a_{2}, 4 ! a_{3}, \ldots\right) B_{n, k}\left(b_{2}, b_{3}, \ldots\right) .
$$

Theorem 3.3 $W_{t}^{(t)}=-i \lambda_{t}$ and $W_{n}^{(t)}=0$ for any $n \neq t$.

Proof ${ }^{1}$ For $k<t$, the Bell polynomial $B_{k, t}(\ldots)$ is zero, hence the sum can be started at $k=1 . B_{k, t}\left(1,2 ! a_{1}, 3 ! a_{2}, \ldots\right)=k !\left[\rho^{k}\right] \phi^{t}(\rho)$ is by Eq. 1.3 the $k^{\text {th }}$ coefficient of the power series $\phi^{t}(\rho)$. Using Lemma 2.5, $\rho(\phi)=\sum_{j=1}^{\infty} \frac{b_{j+1}}{j !} \phi^{j}$, the tree sum $W_{n}^{(t)}$ by Eq. 1.3 can be interpreted as the coefficient of $\phi^{n}$ of a power series

$$
\begin{aligned}
W_{n}^{(t)} & =-i \lambda_{t} \sum_{k=t}^{n} k !\left[\rho^{k}\right] \phi^{t}(\rho) \cdot B_{n, k}\left(b_{2}, b_{3}, \ldots\right) \\
& =-i \lambda_{t}\left[\phi^{n}\right]\left((\phi(\rho(\phi)))^{t}\right)=-i \lambda_{t}\left[\phi^{n}\right] \phi^{t}=-i \lambda_{t} \delta_{n t} .
\end{aligned}
$$

Hence the tree sum is $-i \lambda_{t}$ for $n=t$ and zero otherwise.

If, according to Eq. 3.4, several different interaction terms are present in the original theory, then each of them comes with a different coupling constant $\lambda_{t}$ and a

\footnotetext{
${ }^{1}$ The interpretation of $W_{n}^{(t)}$ as a series coefficient was originally suggested by Ali Assem Mahmoud. This simplified the proof considerably.
} 
corresponding tree sum $W_{n}^{(t)}$. Each tree which contains at least two different interaction vertices $w_{j}^{\left(t_{1}\right)}$ and $w_{k}^{\left(t_{2}\right)}$ can be decomposed into a product of trees containing only a single one, connected via an intermediate propagator. This is always possible since an interaction vertex $w_{i}^{t}$ cannot cancel an adjacent propagator, so there is always (at least) one uncancelled propagator between any two interaction vertices. The total tree sum linear in the coupling constants consequently is a sum of the individual contributions

$$
W_{n}:=\sum_{t=3}^{\infty} W_{n}^{(t)}=-i \sum_{t=3}^{n} \lambda_{t} \sum_{k=t}^{n} B_{n, k}\left(b_{2}, b_{3}, \ldots\right) B_{k, t}\left(1,2 ! a_{1}, 3 ! a_{2}, 4 ! a_{3}, \ldots\right) .
$$

From Theorem 3.3 one has

$$
W_{n}=-i \lambda_{n} \quad \forall n \geq 3
$$

\subsection{Feynman Rules of the Connected Perspective}

This section generalizes Section 2.6 to diffeomorphisms of an underlying interacting theory.

First, (1) of Lemma 2.10 needs to be modified for an underlying interacting theory:

Lemma 3.4 A n-valent metavertex with all external edges onshell has amplitude $W_{n}=-i \lambda_{n}$.

Proof By Eq. 3.9, $W_{n}$ is a tree sum which evaluates to a polynomial in the external momenta (this polynomial being 1), hence it is a metavertex Definition 2.8. Since $W_{n}$ already represents the sum of all trees, there is no other metavertex with the claimed properties.

Note that this metavertex $W_{n}$ vanishes in the free diffeomorphism case (i.e. $\lambda_{n}=$ 0) by Lemma 2.7, hence Lemmas 2.10 and 3.4 are consistent.

In the connected perspective of an underlying interacting theory there are two different metavertices: $V_{n}$ arising from the diffeomorphism and $W_{n}$ arising from the original interaction. Lemma 2.12 continues to hold for edges between two $V_{n}$-type metavertices. The $W_{n}$-type metavertex does not cancel any edge, so the only remaining possiblity is a $V_{n}$-metavertex cancelling an edge joining it to an $W_{n}$-metavertex.

Lemma 3.5 In the connected perspective, a metavertex $V_{m}$ must not cancel an internal edge adjacent to a $W_{t}$ metavertex.

Proof We have $W_{t}=-i \lambda_{t}$ by Theorem 3.3. Let $T$ be a tree where an $V_{m}$ metavertex cancels an edge connecting it to a $W_{t}$ metavertex. The result of this cancellation is a tree with $m+t-2$ external edges, all of which can be choosen onshell, and no internal propagators left. Sum over all permutations of external edge and all choices 
$m, t$ leading to the same number of external edges, then this sum reproduces $W_{t+m-2}$ with amplitude $-i \lambda_{t+m-2}$ by Theorem 3.3, i.e. it has no contribution proportional to $\lambda_{t}$. All terms $\propto \lambda_{t}$ add up to zero and can thus be left out.

Lemma 3.5 means that all trees where a metavertex $V_{m}$ cancels an edge adjacent to a metavertex $W_{t}$ eventually do not contribute and can thus be left out from the start. Consequently, in the connected perspective, the cancellation of such an internal edge will be forbidden.

Definition 3.6 (Tree sum $A_{n}^{\prime}{ }_{n}$ ) $A^{\prime}{ }_{n}^{j}$ is defined as the sum of all trees with $n$ external edges, $j$ of which are offshell, in the diffeomorphism of the interacting theory (3.1).

$A^{\prime}{ }_{n}^{j}$ is the generalization of $A_{n}^{j}$ from Definition 2.6 to include also interaction vertices. The relation between Definition 3.6 and $W_{n}$ from Definition 3.1 is

$$
W_{n}^{(t)}=\left.A_{n}^{\prime 0}\right|_{\text {only terms linear in } \lambda_{t}} .
$$

Compare also Fig. 7 for the relation between $A_{n}^{\prime j}, A_{n}^{j}$ and $b_{n}^{\prime}$ (to be definied in Definition 3.8).

We summarize the Feynman rules for the connected perspective of an underlying interacting field analogous to Theorem 2.17:

Theorem 3.7 (Feynman rules of the connected perspective of an interacting field diffeomorphism) Assuming the vanishing of tadpoles, the n-point connected amplitude is obtained by summing over all connected graphs $\Gamma$ such that

(1) Each internal edge e contributes a propagator factor $\frac{i}{s_{e}}$.

(2) There are two types of $(k>2)$-valent metavertices, $V_{k}$ and $W_{k}=-i \lambda_{k}$.

(3) $V_{k}=-i b_{k}\left(s_{1}+s_{2}+\ldots+s_{k}\right)$ where keeping a summand $s_{e}$ in this amplitude amounts to cancelling the adjacent edge $e$.

(4) The metavertices $V_{k}$ do not cancel internal edges of $\Gamma$, each metavertex $V_{k}$ is adjacent to at least one external offshell edge.

Proof Combination of Eq. 2.4 and Lemmas 2.12, 2.15, 2.16. 3.4 and 3.5. The role of tadpoles for (2) and (4) in loop-level graphs will be discussed in Section 3.5.

\subsection{Offshell Treelevel Amplitudes}

Definition 3.8 (Tree sum $b_{n}^{\prime}$ ) $b_{n}^{\prime}$ is the sum of all trees with $n$ external edges, $n-1$ of them onshell and without propagator, and one external edge offshell with corresponding propagator. $b_{n}^{\prime}$ consists of both pure diffeomorphism vertices $i v_{j}$ and interaction-diffeomorphism vertices $-i w_{j}^{(t)}$.

To construct $b_{n}^{\prime}$, we fix one of the external edges to be the offshell one - call it number $n$ - and include the propagator $\frac{i}{s_{n}}$. 
Lemma 3.9 In the connected perspective, two different types of trees contribute to $b_{n}^{\prime}$ :

(1) Trees without any metavertex $V_{k}$, i.e. consisting of metavertices $W_{k}=-i \lambda_{k}$ exclusively. These are precisely those trees which would make up $b_{n}^{\prime}$ if there were no diffeomorphism at all, they consist of the vertices of the underlying interacting theory.

(2) Trees with a single metavertex $V_{k}$ for $k \leq n$, cancelling the external offshell edge. If $k<n$ then a sufficient number of vertices $W_{j}$ is attached to $V_{k}$ via un-cancelled internal edges.

Proof By Theorem 3.7, $b_{n}^{\prime}$ must be built from trees made of the two available types of metavertices, $V_{k}$ and $W_{k}$, with suitable valences $k$. But $V_{k}$ must be adjacent to an external offshell edge, consequently, there is only one position in the tree where an $V_{k}$-metavertex can possibly reside. This gives rise to the two contributions as claimed.

Lemma 3.9 implies that in a diffeomorphism of $\phi^{t}$-theory (where $W_{j \neq t}=0$ by Eq. 3.9), all tree sums $b_{n}^{\prime}$ with $n<t$ are equal to the pure diffeomorphism tree sums,

$$
\begin{array}{ll}
b_{n}^{\prime}=b_{n} & \forall n<t \\
b_{n}^{\prime}=b_{n}+\frac{\lambda_{t}}{s_{n}} & \text { if } n=t .
\end{array}
$$

If $n>t$, trees built from both interaction metavertices $W_{t}$ and diffeomorphism metavertices $V_{k}=-i s_{n} b_{k}$ contribute to $b_{n}^{\prime}$. Contrary to $b_{n}$ from Definition 2.2 , the tree sums $b_{n}^{\prime}$ by Lemma 3.9 do depend on external momenta. Consequently, there is no analogue of Theorem 2.4 for $b_{n}^{\prime}$. This was to be expected: Since the diffeomorphism (1.2) ist tangent to identity, it necessarily reproduces the treelevel $n$-point amplitudes of the underlying interacting theory in the limit of the diffeomorphism going to identity. But those do depend on external momenta.

Example 3.10 (Tree sums $b_{n}^{\prime}$ ) Consider a diffeomorphism of $\phi^{3}$-theory, i.e. $t=3$ and compute $b_{n}^{\prime}$ (Definition 3.8). The offshell edge ist taken to be number $n$, momentum conservation implies $s_{n}=s_{1+\ldots+(n-1)}$.

$$
\begin{aligned}
b_{3}^{\prime}=b_{3}+ & \left(-i \lambda_{3}\right) \frac{i}{s_{1+2}}=b_{3}+\frac{\lambda_{3}}{s_{1+2}} \\
b_{4}^{\prime}=b_{4}+ & \left(b_{3}+\frac{\lambda_{3}}{s_{1+2+3}}\right)\left(-i \lambda_{3}\right)\left(\frac{i}{s_{2+3}}+\frac{i}{s_{1+3}}+\frac{i}{s_{1+2}}\right) \\
b_{5}^{\prime}=b_{5}+ & b_{4}\left(-i \lambda_{3}\right)\left(\frac{i}{x_{1+2}}+5 \text { more }\right) \\
& +\left(b_{3}+\frac{\lambda_{3}}{s_{1+2+3+4}}\right)\left(-i \lambda_{3}\right)^{2}\left(\frac{i^{2}}{s_{1+2} s_{3+4}}+2 \text { more }\right) \\
& +\left(b_{3}+\frac{\lambda_{3}}{s_{1+2+3+4}}\right)\left(-i \lambda_{3}\right)^{2}\left(\frac{i^{2}}{s_{1+2} s_{1+2+3}}+5 \text { more }\right)
\end{aligned}
$$


These terms can be interpreted in terms oft trees built according to Lemma 3.9 as shown in Fig. 6. Note that the upper vertex $V_{k}=-i s_{n} b_{k}$ becomes $b_{k}$ by multiplication with the propagator $\frac{i}{s_{n}}$.

Comparable to Eq. 2.5, the tree sum $b_{n}^{\prime}$ from Definition 3.8 equals $A_{n}^{\prime 1}$ from Definition 3.6 up to the symmetrization of the offshell edge and an included propagator. But since $b_{n}^{\prime}$ depends on momenta, the symmetrization involves an actual sum over different orientations of $b_{n}^{\prime}$, not just over the offshell variables $s_{e}$ like in Eq. 2.5,

$$
A_{n}^{\prime 1}=-i \sum_{\text {Perm. }} b_{n}^{\prime} s_{n} \neq-i b_{n}^{\prime} \cdot\left(s_{1}+\ldots+s_{n}\right) .
$$

Lemma 3.11 The tree sum ${A^{\prime}}_{n}$ from Definition 3.6 coincides with the tree sum with $n$ external edges onshell of the underlying interacting theory. That is, the diffeomorphism does not alter the S-matrix at treelevel.

Proof Follows from Theorem 3.7 part (4): ${A^{\prime}}_{n}^{0}$ has no external offshell edges, consequently no metavertex of $V_{k}$-type contributes to it. All that remains is a sum over trees built from $W_{k}$-vertices, i.e. the sum over the trees of the underlying interacting theory.

Alternatively, the same conclusion can be drawn from Lemma 3.9 or Eq. 3.12 if one amputates the external offshell edge via multiplication with $-i s_{n}$ and takes the onshell limit $s_{n} \rightarrow 0$.

By Theorem 3.7, treelevel amplitudes with more than one external edge offshell in the connected perspective can contain more than one metavertex $V_{k}$. There are

$$
b_{3}^{\prime}=\mathbf{x}=\mathbf{R}
$$
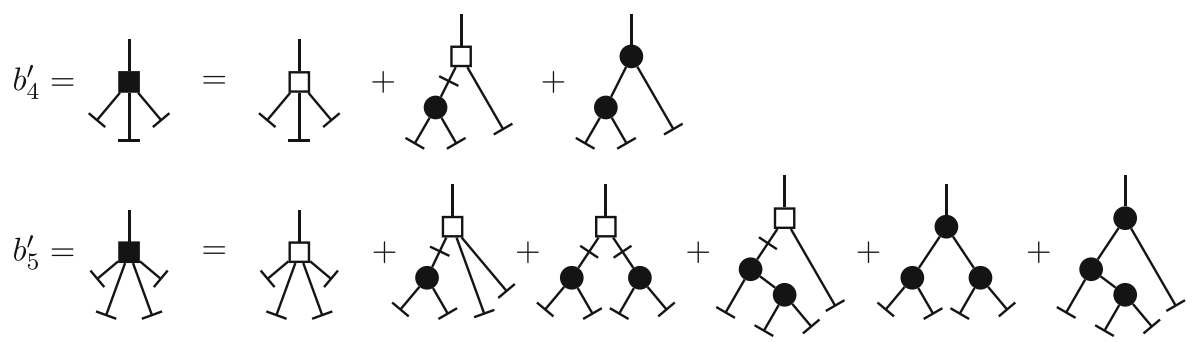

Fig. 6 Construction of $b_{n}^{\prime}$ for $t=3$ (a diffeomorphism of $\phi^{3}$-theory) from Example 3.10 as a sum of metavertices $W_{3}=-i \lambda_{3}$ and zero or one metavertex $V_{k}$. The sum over permutations of the lower edges is not indicated. Note that perpendicular lines have no meaning for an interaction metavertex $W_{n}$ as it never cancels an adjacent edge anyway 
$\left.A_{4}^{\prime 4}\right|_{s_{1}=s_{2}=s_{3}=0, \text { propagator }}=b_{4}+\left(b_{3}+\frac{\lambda_{3}}{s_{4}}\right)\left(-i \lambda_{3}\right)\left(\frac{i}{s_{1+2}}+\frac{i}{s_{1+3}}+\frac{i}{s_{2+3}}\right)=b_{4}^{\prime}$.

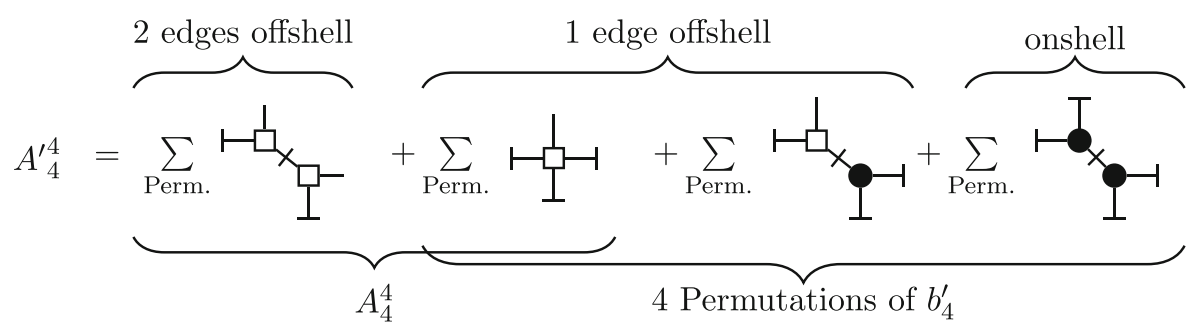

Fig. 7 Construction of $A_{4}^{\prime 4}$ according to Example 3.12. Here, "Perm." indicates a symmetric sum over not only the onshell, but all external edges. Curly brackets show the relationship to the pure diffeomorphism amplitude $A_{4}^{4}$ from Example 2.18 and the tree sum $b_{4}^{\prime}$ from Example 3.10. The onshell part or $A_{4}^{\prime 4}$ coincides with the underlying $\phi^{3}$-theory as expected from Lemma 3.11

uncancelled internal edges between such metavertices as discussed for the free diffeomorphism in Section 2.5. The only difference is that in the present case, an arbitrary number of interaction vertices $W_{k}$ can be present in the graph.

Example 3.12 (Offshell tree sum $A_{4}^{\prime 4}$ ) We compute $A_{4}^{\prime 4}$ from Definition 3.6 for an underlying $\phi^{3}$-theory, $t=3$.

$$
A_{4}^{\prime 4}=A_{4}^{4}+\left(-i b_{3}\left(s_{1}+s_{2}+s_{3}+s_{4}\right)-i \lambda_{3}\right)\left(-i \lambda_{3}\right)\left(\frac{i}{s_{1+2}}+\frac{i}{s_{1+3}}+\frac{i}{s_{1+4}}\right) .
$$

The four contributing trees in the connected perspective are shown in Fig. 7. $A_{4}^{4}$ is the 4-point treelevel amplitude of the free diffeomorphism computed in Example 2.18. Setting $s_{1}=s_{2}=s_{3}=0$ and including the external propagator $\frac{i}{s_{4}}$ turns $A^{\prime \prime 4}$ into $b_{4}^{\prime}$ as computed in Example 3.10:

$$
\left.A^{\prime 4}\right|_{s_{1}=s_{2}=s_{3}=0, \text { propagator }}=b_{4}+\left(b_{3}+\frac{\lambda_{3}}{s_{4}}\right)\left(-i \lambda_{3}\right)\left(\frac{i}{s_{1+2}}+\frac{i}{s_{1+3}}+\frac{i}{s_{2+3}}\right)=b_{4}^{\prime} .
$$

\subsection{Loop Amplitudes}

The behaviour of loop amplitudes for the interacting diffeomorphism is a straightforward generalization of the free diffeomorphism discussed in Section 2.7. Especially, we again require vanishing of tadpole graphs in the connected perspective to maintain consistency of the metavertices.

The only difference of the present case compared to free diffeomorphism is the presence of a second type of metavertex, $W_{n}=-i \lambda_{n}$ originating from the underlying interaction. Following Theorem 3.7, there is no restriction regarding the position of such metavertices in a graph in the connected perspective. Especially, they can appear as internal vertices (Definition 2.13). A metavertex $V_{k}$ on the other hand is always adjacent to at least one of the external edges of the graph, and cancels this edge. 
Consequently, if none of the external edges are offshell, the only type of metavertex present in the connected perspective is $W_{k}$, i.e. the connected perspective reduces to connected graphs of the underlying interacting theory.

To conclude Section 3, we store this result in a theorem:

Theorem 3.13 Consider a diffeomorphism of a polynomially self-interacting scalar field (3.4). Let $F_{m}^{(l)}$ be the sum of all connected (not only 1PI) l-loop Feynman amplitudes of this diffeomorphism with $m$ external edges, all of which are onshell. Assume tadpole graphs vanish. Then $F_{m}^{(l)}$ coincides with the corresponding amplitude of the underlying theory, i.e. the diffeomorphism does not alter the $S$-matrix.

Proof Note that $F_{m}^{(0)}=A_{m}^{\prime 0}$ is the treelevel onshell $m$-point-amplitude which was shown to coincide with the underlying interacting theory in Lemma 3.11.

Compute $F_{m}^{(l)}$ in the connected perspective Section 2.4. Following Section 2.7 and especially Example 2.21, vanishing of tadpole graphs ensures that there is no other type of metavertex than $W_{k}=-i \lambda_{k}$ and $V_{k}$. Then, Feynman rules even for loopamplitudes are given by Theorem 3.7. By (4) of this theorem, a $V_{k}$-type metavertex is adjacent to at least one external offshell edge. $F_{m}^{(l)}$ has no external offshell edges, consequently it consists of $W_{k}$-type metavertices exclusively. These are the vertices of the underlying interacting theory.

Theorem 3.13 is the graph-theoretic version of the result found in [8]. The key mechanism is very similar: A diffeomorphism gives rise to contributions proportional to the offshell-variables of the correlation functions under consideration. If all momenta are onshell, all diffeomorphism contributions obtain a zero prefactor.

As a consequence of Theorem 3.13, one can view all quantum field theories as physically equivalent if they are related by a diffeomorphism. It is then possible to choose out of this equivalence class one particular representative with desirable properties.

\section{Cancellation of Offshell Quantum Corrections}

In this section, we restrict to only one interaction monomial $\phi^{t}$ in the underlying Lagrangian Eq. 3.1. Regardless of the identical $S$-matrix (Theorem 3.13), a diffeomorphism of such a theory alters the offshell correlation functions. The goal is to fix the diffeomorphism in such a way that these altered offshell correlation functions of an interacting theory coincide with the ones of a free scalar quantum field theory. As mentioned in [17], this is especially interesting for the 2-point function, i.e. the propagator: Canonical quantization relies on the assumption that in infinite future and past, the states of an interacting theory asymptotically converge to free field states (e.g. [22, Sec. 1.4.2] or assumption 3 of [18]). These states are then identified with particles observed in scattering experiments which allows to predict experimental outcomes through the LSZ-formalism [18]. On the other hand, if any quantum field theory has the same 2-point-function as a free field, or is unitarily equivalent to a free 
field, this theory is itself a free field [5, 10, 14, 24]. See also [15] for an overview of the different no-go theorems regarding this phenomenon.

By Theorem 3.13, diffeomorphisms of a scalar quantum field theory are physically equivalent even if they are not unitarily equivalent. It might be possible to construct with the help of diffeomorphisms some new notion of asymptotic states which does not violate theorems of axiomatic quantum field theory. We do not pursue such a construction here, but we derive a diffeomorphism with suitable properties.

\subsection{Tree Level}

We consider treelevel amplitudes with one external edge offshell, $b_{n}^{\prime}$ (Definition 3.8) in the connected perspective. We fix an offshell 4-momentum $p$ and let

$$
s_{p}=p^{2}-m^{2} \neq 0
$$

be the offshell variable (Definition 2.1) of the tree sums $b_{n}^{\prime}$. Our goal is to find a diffeomorphism such tat all $b_{n}^{\prime}$ vanish for this specific momentum. Remember that there is only one valence of interaction metavertex, $W_{t}$, since we fixed the interaction to be of $\phi^{t}$-type.

Lemma 4.1 Consider underlying $\phi^{t}$-typ interaction. If

$$
b_{t}=-\frac{\lambda_{t}}{s_{p}} \quad \text { and } \quad b_{k}=0 \forall k \neq t
$$

then $b_{n}^{\prime}=0 \forall n>2$.

Proof Follows from Lemma 3.9. For $1<n<t$, setting $b_{n}=0$ amounts to $b_{n}^{\prime}=0$ as noted in Eq. 3.11. For $n=t$, the given choice of $b_{t}$ makes $b_{t}^{\prime}$ vanish. This choice also implies that the $t$-valent metavertex $V_{t}$ and the $t$-valent interaction vertex $W_{t}$ add up to zero if they appear adjacent to the offshell edge. Setting $b_{k}=0 \forall k \neq t$ implies by Eq. 2.6 that the metavertex $V_{k}=0 \forall k \neq t$.

Now consider any tree $T$ contributing to $b_{n}^{\prime}$. By Lemma 3.9, two situations are possible with regard to the metavertex $v$ adjacent to the offshell edge: Either $v=V_{k}$ for some $k \neq t$, but then $v=0$. Or $v$ has valence $t$, then $v=V_{t}$ or $v=W_{t}$. Since $b_{n}^{\prime}$ is the sum over all trees, one can combine both $t$-valent metavertices into a single "effective" one, $v=V_{t}+W_{t}$. This sum is zero by the first part of the proof. Consequently, in the sum of all trees $T$, any vertex adjacent to the external edge has Feynman amplitude zero and hence the sum vanishes, $b_{n}^{\prime}=0$.

Theorem 4.2 Let $t \geq 3$ be an integer and $\phi$ a scalar quantum field with mass $m \geq 0$ and $\phi^{t}$-type interaction according to Eq. 3.1. Let $A_{n}^{\prime}{ }_{n}^{j}$ be as in Definition 3.6. Assume all offshell external edges of $A_{n}^{\prime j}$ have the fixed offshell variable $s_{p}$. Then the quantum field $\rho$ defined by

$$
\rho(x)=\phi(x)-\frac{\lambda_{t}}{(t-1) ! s_{p}} \phi^{t-1}(x)
$$


has the same tree level amplitudes as a free quantum field with mass $m$, i.e.

$$
\begin{aligned}
& {A_{2}^{\prime 2}}_{2}=-i s_{p} \\
& A_{n}^{\prime j}=0 \forall n>2, j>0 .
\end{aligned}
$$

Proof Tree sums $A_{n}^{\prime}{ }_{n}$ are obtained in the connected perspective by Theorem 3.7 from trees consisting of external (Definition 2.13) metavertices $V_{k}$ and external or internal metavertices $W_{k}$. By assumption, the former are connected to an external edge with offshell variable $s_{p}$, hence they give an amplitude $-i s_{p} b_{k}$ by Eq. 2.5 . Following Lemma 4.1, they are either zero or they cancel against a $W_{t}$-metavertex at the same position, consequently, $A^{\prime}{ }_{k}^{j}=0$ if the conditions of Lemma 4.1 are met. By Lemma 2.5, the coefficients $b_{k}$ are series coefficients of the inverse diffeomorphism

$$
\rho(x)=\sum_{n=1}^{\infty} \frac{b_{n+1}}{n !} \phi^{n}(x)=\frac{b_{2}}{1 !} \phi(x)+\frac{b_{t}}{(t-1) !} \phi^{t-1}(x) .
$$

Note that fixing $s_{p}$ does not fix the 4-vector $p$ completely. A kinematical configuration of $A_{n}^{\prime j}$ where all external edges have offshell variables either zero or $s_{p}$ is in principle possible in terms of overall momentum conservation.

Lemma 4.3 The diffeomorphism $\phi(\rho)$ defined in Theorem 4.2 has the coefficients

$$
\begin{aligned}
3 a_{t-2} & =\frac{\lambda_{t}}{(t-1) ! s_{p}} & & \\
a_{j \cdot(t-2)} & =\left(a_{t-2}\right)^{j} \cdot F_{j}(t-1,1), & & j \in \mathbb{N} \\
a_{k} & =0 & & k \notin \mathbb{N} \cdot(t-2) .
\end{aligned}
$$

Here, $F_{j}(s-1,1)$ are the Fuss-Catalan numbers (1.7).

Proof By Eq. 3.11, $b_{n}^{\prime}=b_{n}$ for $n<t$. By Theorem 2.4, $b_{n}$ is a polynomial in $a_{1}, \ldots, a_{n-2}$. Hence if $a_{k}=0 \forall k<t-2$ then $b_{n}^{\prime}=0 \forall n<t$ and $b_{t}=-(t-1) ! a_{t-2}$. This together with Lemma 4.1 fixes the claimed value of $a_{t-2}=: \alpha$.

Now define a function $C(t)$ such that $\phi(\rho)=\rho \cdot C\left(\alpha \rho^{t-2}\right)$. Insert this function for $\phi$ into Theorem 4.2 to obtain

$$
\alpha \rho^{t-2} \cdot C^{t-1}\left(\alpha \rho^{t-2}\right)+1=C\left(\alpha \rho^{t-2}\right) .
$$

The generating function of the Fuss-Catalan-Numbers (1.7), $C_{a, b}(y)=$ $\sum_{m=0}^{\infty} y^{m} F_{m}(a, b)$, fulfills the functional equation [21, theorem. 2.1]

$$
C_{a, b}(y)=\left(y C_{a, b}^{\frac{a}{b}}(y)+1\right)^{b} .
$$

Thus $C(t)$ is the generating function of Fuss-Catalan-Numbers $\left\{F_{j}(t-1,1)\right\}_{j}$, with argument $y=\alpha \rho^{t-2}$. Taking coefficients and identifying (1.2) yields the values of $a_{k}$ for $k>t-2$. 


\subsection{Loop Amplitudes}

By construction, the connected amplitudes of the diffeomorphism Theorem 4.2 are built in the connected perspective from two metavertices, $W_{t}$ and $V_{t}$. All metavertices with valence other than $t$ vanish. Assume that tadpole graphs vanish, then by Sections 2.7 and 3.5 the Feynman rules Theorem 3.7 of the connected perspective remain intact even for loop graphs, i.e. no other types of metavertices arise.

Consequently, the proof of Theorem 4.2 holds also for loop amplitudes as long as there is at least one offshell external edge with momentum $s_{p} \neq 0$ and all remaining external edges are onshell. Therefore, the diffeomorphism given by Theorem 4.2 and Lemma 4.3 indeed mimics a free field in the following sense: There exists one specific offshell variable $s_{p}$ such that any $n>2$-point-correlation function vanishes if at least one external offshell variable is $s_{p}$ and all remaining edges are onshell. Furthermore, the 2-point-correlation function equals $-i s_{p}$ at this offshell variable $s_{p}$.

\subsection{Multiple Interaction Terms}

We note in passing that a similar diffeomorphism as Theorem 4.2 is possible even if there is more than one interaction monomial in the underlying Lagrangian. In that case, Lemma 4.1 has to be changed such that $b_{t}=-\lambda_{t} s_{p}^{-1}$ for each $t$ where $\lambda_{t} \neq 0$. This is always possible because all $\left\{b_{k}\right\}_{k>2}$ can be choosen independent from each other.

\section{Scalar Field with Arbitrary Propagator}

As suggested below Lemma 2.23, the amplitude of the tree sums $b_{n}$ (Definition 2.2) does not depend on the actual form of the propagator. In this section, we examine in what sense the results of Sections 2 and 3 continue to hold if the free part of the underlying Lagrangian is changed, i.e. if it contains higher than second derivatives. Lagrangians involving higher derivatives have for example been studied in the context of fixed points in more than four spacetime dimensions [9] or conformal field theory [3], especially conformal gravity [19, 26]. Such theories can be unitary $[20,25]$.

\subsection{Generalized Free Lagrangian Density}

A scalar field is free if the Lagrangian is quadratic in the field variable $\phi$ and its derivatives. Lorentz invariance dictates that any derivative $\partial_{\mu} \phi$ be contracted with $\partial^{\mu} \phi$. By partial integration, all derivatives can be concentrated on one of the two field variables up to a vanishing total derivative. Hence a general free Lagrangian has the form

$$
\mathcal{L}_{\phi}=\frac{1}{2} \phi(x) \hat{S} \phi(x)
$$


where $\hat{S}$ is a linear Lorentz invariant differential operator

$$
\hat{S}=\beta_{0}+\beta_{1}\left(-\partial_{\mu} \partial^{\mu}\right)+\beta_{2}\left(-\partial_{\mu} \partial^{\mu}\right)^{2}+\ldots=\sum_{k=0}^{\infty} \beta_{k}\left(-\partial_{\mu} \partial^{\mu}\right)^{k}
$$

$\left\{\beta_{j}\right\}_{j}$ are spacetime-independent constants. The ordinary free field (1.1) amounts to $\beta_{0}=-m^{2}, \beta_{1}=1, \beta_{j>1}=0$. The classical field defined by Eq. 5.1 has the equation of motion

$$
\hat{S} \phi(x)=0
$$

which is linear as desired for a free field. In momentum space, the operator $\hat{S}$ acts as multiplication,

$$
\hat{S} \phi(x)=\int \frac{\mathrm{d}^{4} k}{(2 \pi)^{4}} \underbrace{\left(\beta_{0}+\beta_{1} k_{\mu} k^{\mu}+\beta_{2} k_{\mu} k^{\mu} k_{\nu} k^{\nu}+\ldots\right)}_{=: S_{k}} \phi(k) e^{-i k x} .
$$

The propagator of the field $\phi$ is defined as the inverse of the field differential operator Eq. 5.2 (i.e. as a Green's function) in momentum space,

$$
\Gamma_{2, \text { free }}^{-1}(k)=\langle\phi(k) \phi(-k)\rangle=\frac{i}{S_{k}} .
$$

With regards to the propagator, the quantity

$$
S_{k}=\beta_{0}+\beta_{1} k^{2}+\beta_{2} k^{4}+\ldots=\sum_{n=0}^{\infty} \beta_{n}\left(k^{2}\right)^{n}
$$

replaces the offshell variable $s_{k}=-m^{2}+k^{2}$ from Definition 2.1. Consequently, in the following, the term onshell will refer to $S_{k}=0$ regardless if $s_{k}=0$. We do not care here if the pole $S_{k}=0$ of the propagator (5.3) corresponds to a one-particle state (i.e. is a simple pole with unit residue) as in the standard interpretation of perturbative quantum field theory.

\subsection{Feynman Rules of the Ordinary Perspective}

Applying the diffeomorphism Eqs. 1.2 to 5.1 gives rise to the Lagrangian

$$
\mathcal{L}_{\rho}=\frac{1}{2} \sum_{j=0}^{\infty} \sum_{k=0}^{\infty} a_{j} a_{k} \rho^{j+1} \hat{S} \rho^{k+1} .
$$

Since $a_{0}=1$, the propagator of $\rho$ coincides with that of $\phi$ from Eq. 5.3. To derive the vertex Feynman rules of $\rho$, consider the Fourier transform of the action of $\hat{S}$ on a product of fields.

$$
\hat{S} \phi^{n}(x)=\int \cdots \int \frac{\mathrm{d}^{4} k_{1}}{(2 \pi)^{4}} \cdots \frac{\mathrm{d}^{4} k_{n}}{(2 \pi)^{4}} \phi\left(k_{1}\right) \cdots \phi\left(k_{n}\right) S_{1+\ldots+n} \cdot e^{-i k_{1} x} \cdots e^{-i k_{n} x} .
$$


We have introduced $S_{1+\ldots+n}$ analogous to Definition 2.1,

$$
S_{1+2+\ldots+n}=\sum_{j=0}^{\infty} \beta_{j}\left(k_{1}+k_{2}+\ldots+k_{n}\right)^{2 j} .
$$

The $n$-valent diffeomorphism vertex arises from terms $\phi^{j} \hat{S} \phi^{k}$ in Eq. 5.5 where $j+$ $k=n$. Summing over all permutations of $n$ edges, this amounts to $j$ ! times a sum over all ways to choose $k$ out of $n$, each of them multiplied by $k$ ! because $S_{1+\ldots+k}$ is symmetric under permutation of its indices. Hence the vertex Feynman rule is

$$
i v_{n}=i \frac{1}{2} \sum_{k=1}^{n-1} a_{n-k-1} a_{k-1}(n-k) ! k ! \sum_{P \in Q^{(n, k)}} S_{P} .
$$

The latter sum consists of all $\left|Q^{(n, k)}\right|=\left(\begin{array}{l}n \\ k\end{array}\right)$ possibilities to choose $k$ out of $n$ external edges without distinguishing the order,

$$
Q^{(n, k)}:=\left\{\left\{j_{1}, \ldots, j_{k}\right\} \subseteq\{1, \ldots, n\}\right\} .
$$

These are the same partitions which make up elementary symmetric polynomials, i.e. the elementary symmetric polynomial of degree $k$ in $n$ variables is $e_{k}\left(\left\{z_{1}, \ldots, z_{n}\right\}\right)=$ $\sum_{P \in Q^{(n, k)}} \prod_{z \in P} z$.

Example 5.1 (Choosing 3 out of 5) For $n=5, k=3$ there are ten summands

$$
\begin{array}{r}
\sum_{P \in Q^{(5,3)}} S_{P}=S_{1+2+3}+S_{1+2+4}+S_{1+2+5}+S_{1+3+4}+S_{1+3+5} \\
+S_{1+4+5}+S_{2+3+4}+S_{2+3+5}+S_{2+4+5}+S_{3+4+5} .
\end{array}
$$

The indices correspond to the summands of the elementary symmetric polynomial

$$
e_{3}\left(\left\{z_{1}, \ldots, z_{5}\right\}\right)=z_{1} z_{2} z_{3}+z_{1} z_{2} z_{4}+(6 \text { more })+z_{2} z_{4} z_{5}+z_{3} z_{4} z_{5} .
$$

Momentum is conserved at each vertex, $p_{1}+\ldots+p_{n}=0$, hence only the elements of $Q^{(n, k)}$ for $k \leq \frac{n}{2}$ are linearly independent. For $k>\frac{n}{2}$, each $S_{P}$ coincides with a term which was already present, this mechanism eventually cancels the prefactor $\frac{1}{2}$ in the Feynman rule (5.6).

Example 5.2 (Vertices) Using $S_{1+2} \equiv S_{3}$ etc., the 3-valent vertex (5.6) is

$$
\begin{aligned}
i v_{3} & =i \frac{1}{2} a_{1} 2 ! 1 !\left(S_{1}+S_{2}+S_{3}\right)+i \frac{1}{2} a_{1} 1 ! 2 !\left(S_{1+2}+S_{1+3}+S_{2+3}\right) \\
& =2 i a_{1}\left(S_{1}+S_{2}+S_{3}\right) .
\end{aligned}
$$

This result appears to coincide with Fig. 1, but this is coincidence. Already the next vertex is

$$
i v_{4}=6 i a_{2}\left(S_{1}+S_{2}+S_{3}+S_{4}\right)+4 i a_{1}^{2}\left(S_{1+2}+S_{1+3}+S_{2+3}\right) .
$$

Equation 5.6 does not generally reduce to Eq. 2.2 because for higher than second powers, the sum of momenta does not decompose, i.e. $S_{1+2}$ cannot generally be expressed as $S_{1}, S_{2}$ and $m^{2}$. 


\subsection{Feynman Rules of the Connected Perspective}

The vertex Feynman rule (5.6) involves summands $S_{j}$ which directly cancel an adjacent propagator $\frac{i}{S_{j}}$, but also summands $S_{j+k+\ldots}$. This cancellation suggests to once again switch to the connected perspective (2.4).

The diffeomorphism of an arbitrary free Lagrangian (5.1) gives rise to Feynman rules (5.6) seemingly quite different than that of the ordinary free theory (2.2). We need to establish the fact that even in the present case, the tree sums $A_{n}^{1}$ defined in 2.6 (built from vertices (5.6) instead of Eq. 2.2) act as metavertices Definition 2.8 in the connected perspective.

Lemma 5.3 Let e be an internal edge in a tree sum $A_{n}^{n}$ from Definition 2.6. Then there is no summand proportional to $S_{e}$ in $A_{n}^{n}$.

Proof Consider one arbitrary tree $F \in A_{n}^{n}$. Since $e$ is internal, it is adjacent to two vertices. Let their valence be $j$ resp. $k$, then $e$ together with the two vertices form a subtree $T \subseteq F$ which has $j+k-2 \leq n$ external edges, some of which may be external edges of $A_{n}^{n}$. Its amplitude is

$$
T=i v_{j} \cdot \frac{i}{S_{e}} \cdot i v_{k},
$$

We identify the external edges of $T$ with numbers $1, \ldots, j+k-2$ and consider terms proportional to $S_{e}$ in $T$. Such proportionality requires both vertices $v_{j}, v_{k}$ to cancel the edge $e$ connecting them. By Eq. 5.6 this summand has the Feynman amplitude

$$
i a_{k-2}(k-1) ! S_{e} \cdot \frac{i}{S_{e}} \cdot i a_{j-2}(j-1) ! S_{e}=-i a_{j-2} a_{k-2}(j-1) !(k-1) ! S_{e} .
$$

The momentum running through $e$ corresponds to one way to partition the external momenta $\{1, \ldots, j+k-2\}$ of $T$ into two sets of $(k-1)$ resp. $(j-1)$ elements.

The tree sum $A_{n}^{n}$ also contains a tree $F^{\prime}$ where $T \subseteq F$ is replaced by a single vertex $i v_{j+k-2} \subseteq F^{\prime}$ at the same positon, see Fig. 8. By Eq. 5.6 this vertex is made of summands proportional to $S_{P}$ where $P$ is any way to partition the $j+k-2$
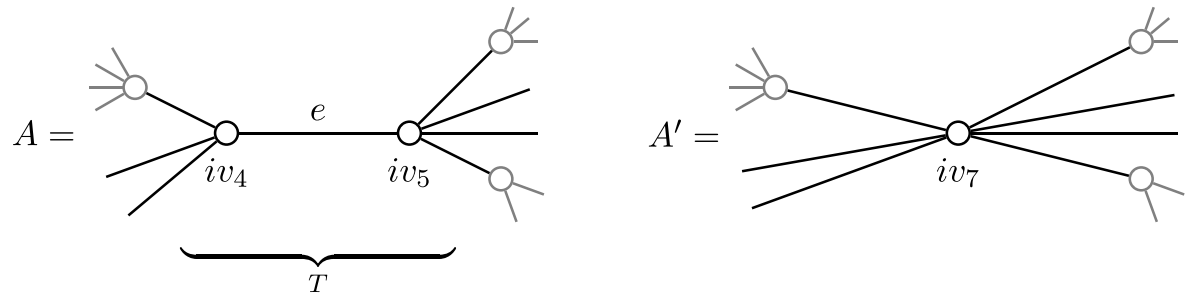

Fig. 8 Illustration of contributions to a tree sum for $j=4, k=5$. A contains a subtree $T$ consisting of one internal edge $e$ connected to two vertices whereas in $A^{\prime}$ that subtree is replaced by a single vertex 
external edges into two sets. Especially, precisely two such partitions resembles the momentum running through $e$ such that $S_{P}=S_{e}$. They contribute to $i v_{j+k-2}$ with an amplitude

$i \frac{1}{2} a_{j-2} a_{k-2}(j-1) !(k-1) ! S_{e}+i \frac{1}{2} a_{k-2} a_{j-2}(k-1) !(j-1) ! S_{e}=i a_{j-2} a_{k-2}(j-1) !(k-1) ! S_{e}$.

This contribution is equal up to sign to the contribution from $T$, i.e. $T+i v_{j+k-2}=0$ and consequently $F+F^{\prime}=0$.

We did not impose any conditions on $T$. Whenever there is an internal edge $e$, the sum $A_{n}^{n}$ contains the same tree where this edge is replaced by a vertex and they conspire to cancel the contribution proportional to $S_{e}$.

The vertex Feynman rules (5.6) allow for a transparent discussion of the cancellation of internal edges in tree sums. Therefore we believe that they are a more intuitive form than Eq. 2.2 even in the case of an ordinary quadratic propagator. Especially, it is nice to see how the Lagrangian being quadratic in $\phi$ (i.e. free) translates to the Feynman rules containing partitions of edges into two subsets. This also suggests an "intuitive" interpretation of the proof of Theorem 3.3: For underlying $\phi^{t}$-theory, the diffeomorphism interaction vertices $-i w_{n}$ (3.5) represent all ways to partition $n$ external edges into $t$ subsets. This is precisely the same partition which arises by glueing tree sums $b_{k_{i}}$ to an original $\phi^{t}$-vertex, see Fig. 5, and once more they cancel each other.

Theorem 5.4 For any $n>2$ the tree sums with one external edge offshell Definition 2.6 are

$$
A_{n}^{1}=-i b_{n} \cdot\left(S_{1}+\ldots+S_{n}\right)
$$

where $b_{n}$ depend only on $\left\{a_{j}\right\}_{j}$ via Theorem 2.4 .

Proof By the Euler characteristic (1.8), a tree $A \in A_{n}^{1}$ with $\left|V_{A}\right|$ vertices contains $\left|V_{A}\right|-1$ internal edges. By Eq. 5.6 each vertex is proportional to some $S$. Each internal edge has an propagator proportional to $\frac{1}{S}$ (these are not the same $S_{k}$, we just count powers of $S$ ). Consequently, $A_{n}^{1}$ is in total proportional to $S$.

By Lemma 5.3 there is no summand $\propto S_{e}$ with any internal edge $e$ of $A_{n}^{1}$. Also, no vertex (5.6) is of higher order than one in $S$ and each external edge is connected to only one vertex in any $A \in A_{n}^{1}$. Therefore there cannot be a summand proportional to $S_{j}^{k>1}$ for an external edge $j$. Further, there are no factors $S_{j}^{-1}$ for external edges $j$ since their propagators are not included in $A_{n}^{1}$. Consequently, an individual tree $A \in A_{n}^{1}$ must be of the form $C_{n} \frac{S_{j} S_{k} \cdots}{S_{e} S_{f} \cdots}$ where $j \neq k \neq \ldots$ are external momenta and $e \neq f \neq \ldots$ internal edges (i.e. partitions of the external momenta) and there is one more factor in the numerator than in the denominator. But in $A_{n}^{1}$, only one of the $n$ external edges is offshell, consequently there can be at most one non-zero factor in the numerator. The only non-zero term contributing to $A$ is of the form $S_{j} \cdot c_{n}$ were $c_{n}$ does not depend on any $S$. Since $S$ 
represent the only possible dependence on kinematics in the Feynman rules (5.6), the factor $c_{n}$ must be independent of kinematics. Symmetrization of the external variable $S_{j}$ produces the claimed form. Consistency of Eq. 2.5 in the case $S_{k} \rightarrow s_{k}$ requires that $C_{n}=b_{n}$ are the known coefficients from Theorem 2.4 resp Lemma 2.22

Replacing $s_{k} \rightarrow S_{k}$, the statements of Section 2.5 regarding computation of offshell tree sums continue to hold. Especially, the Feynman rules of the connected perspective are again Theorem 2.17 with the only difference that the offshell variable $s_{k}$ is to be replaced with $S_{k}$ from Eq. 5.4.

Lemma 5.5 Consider a global diffeomorphism of a free theory with non-standard propagator as given in Eq. 5.1. If tadpoles vanish, then the diffeomorphism does not alter the $S$-matrix.

Proof Compare Lemma 2.7. Treelevel follows from Theorem 5.4 since no summand remains if all $S_{j}=0$. Having established Theorem 2.17, loop amplitudes vanish as discussed in Section 2.7.

\subsection{Interacting Theory}

If instead of Eq. 5.1 the underlying Lagrangian density is

$$
\mathcal{L}_{\phi}=\frac{1}{2} \phi(x) \hat{S} \phi(x)-\frac{\lambda_{t}}{t !} \phi^{t}(x)
$$

additionally the vertices $-i w_{n}^{(t)}$ given by Eq. 3.2 are present. Replacing (2.5) by Theorem 5.4, the combinatoric argument from Section 3.2 still works and Eq. 3.9 holds. Compared to the free theory, the connected perspective of an underlying interacting theory obtains a second class of metavertices $W_{n}=-i \lambda_{n}$ and the $S$-matrix is unaffected by the diffeomorphism.

\section{Non-Diffeomorphism Field Transformations}

In Section 5 we have established that the combinatorics of field diffeomorphisms still works if the propagator contains higher than second derivatives. On the other hand, we noted below Eq. 3.6 that a diffeomorphism does not change the power of the derivatives, i.e. it produces vertices of the same order in momenta as the underlying inverse propagator. In this last section, we demonstrate that it is possible to combine both effects to construct a transformation $\phi \rightarrow \rho$ which turns an ordinary field theory $\phi$ into a diffeomorphism of a higher derivative field $\rho$. We restrict to an underlying free theory. This transformation $\phi \rightarrow \rho$ contains derivatives, it is no longer a diffeomorphism. 


\subsection{Arbitrary Propagator from Field Transformation}

We first consider a transformation involving derivatives, but not a global diffeomorphism, of the form

$$
\phi(x)=\sum_{k=0}^{\infty} \alpha_{k}\left(-\partial_{\mu} \partial^{\mu}\right)^{k} \rho(x) .
$$

Applied to the standard free Lagrangian (1.1), this yields (after $2 k+1$ partial integrations)

$$
\mathcal{L}_{\rho}=\frac{1}{2} \rho(x) \sum_{n=0}^{\infty} \sum_{m=0}^{n} \alpha_{n-k} \alpha_{k}\left(-\partial_{\mu} \partial^{\mu}-m^{2}\right)\left(-\partial_{\mu} \partial^{\mu}\right)^{n} \rho(x) .
$$

This equals the Lagrangian (5.1) with the operator $\hat{S}=\sum_{k=0}^{\infty} \beta_{k}\left(-\partial_{\mu} \partial^{\mu}\right)^{k}$ where

$$
\beta_{n}=\sum_{k=0}^{n-1} \alpha_{n-1-k} \alpha_{k}-m^{2} \sum_{k=0}^{n} \alpha_{n-k} \alpha_{k}
$$

By this, the transformed field $\rho$ can be identified with the underlying field $\phi$ in the free field case of Section 5.

\subsection{Global Diffeomorphism and Non-Local Transformation}

Equation 6.2 suggests that with Eq. 6.3 the non-locally transformed field $\rho$ from Eq. 6.1 takes the role of the underlying field $\phi$ in the global diffeomorphism. The global diffeomorphism (1.2) replaces $\rho$ in Eq. 6.1.

Definition 6.1 In a combined transformation, the field $\phi(x)$ is replaced by

$$
\phi(x)=\sum_{k=0}^{\infty} \alpha_{k}\left(-\partial_{\mu} \partial^{\mu}\right)^{k}\left(\sum_{j=0}^{\infty} a_{j} \rho^{j+1}(x)\right)
$$

where both $\left\{\alpha_{k}\right\}_{k \geq 0}$ and $\left\{a_{j}\right\}_{j \geq 0}$ are constants and $a_{0}=1$.

Indeed, an explicit calculation shows that it is not possible to have $\alpha_{k}$ depend on $j$, i.e. to transform different monomials with different derivative operators. If that were the case, the cancellation property of the global diffeomorphism would break down, it would fail to produce vertices proportional to an inverse propagator.

The transformation Definition 6.1 applied to a free Lagrangian (1.1) gives rise to a field $\rho$ which has propagator (5.3) with $\beta_{n}$ from Eq. 6.3 and ( $n \geq 3$ )-valent interaction vertices (5.6). By Theorem 5.4, all $(n>2)$-valent tree sums vanish in the onshell limit $X_{j}=0 \forall j \in\{1, \ldots, n\}$, thus $\rho$ is a free theory. Note that on the other hand its 2-point function stays (5.3), so the derivative part of the non-local transformation Definition 6.1 has a potential influence on observables. 


\section{Conclusion}

We have continued the perturbative examination of diffeomorphisms of quantum fields started in $[16,17]$ and reached the following results:

(1) It is possible to systematically treat the topology-changes introduced by vertices which cancel adjacent edges. To this end, we developed the connected perspective with Feynman rules given by Theorem 2.17 and 3.7 and generalizations in Section 5.

(2) Invariance of the $S$-matrix under global field diffeomorphisms is a consequence of Feynman rule combinatorics. It does neither depend on a specific form of the propagator (Lemma 5.5) nor is it restricted to the underlying field being free (Theorem 3.13). Thus, scalar quantum field theories related by a diffeomorphism can be regarded physically equivalent.

(3) The coefficients of the diffeomorphism of an interacting theory can be chosen such that for a specific fixed offshell momentum, the interaction is cancelled and the connected amplitudes equal those of a free theory (Theorem 4.2).

(4) In the case of an underlying free Lagrangian, it is possible to extend the global diffeomorphism to a transformation involving derivatives in a way that preserves the combinatoric structure of the global diffeomorphism (Section 6.2).

For points (1) to (3), a crucial requirement is vanishing of tadpole integrals.

Funding Open Access funding provided by Projekt DEAL.

Open Access This article is licensed under a Creative Commons Attribution 4.0 International License, which permits use, sharing, adaptation, distribution and reproduction in any medium or format, as long as you give appropriate credit to the original author(s) and the source, provide a link to the Creative Commons licence, and indicate if changes were made. The images or other third party material in this article are included in the article's Creative Commons licence, unless indicated otherwise in a credit line to the material. If material is not included in the article's Creative Commons licence and your intended use is not permitted by statutory regulation or exceeds the permitted use, you will need to obtain permission directly from the copyright holder. To view a copy of this licence, visit http://creativecommonshorg/licenses/by/4.0/.

\section{References}

1. Apfeldorf, K.M., Camblong, H.E., Ordonez, C.R.: Field redefinition invariance in quantum field theory. In: Modern Physics Letters A 16.03, pp. 103-112 (2001). issn: 0217-7323, 1793-6632, https://doi.org/10.1142/S021773230100319X. arXiv:0003287

2. Brown, F., Kreimer, D.: Angles, scales and parametric renormalization. In: arXiv:1112.1180 [hep-th] (visited on 03/08/2019) (2011)

3. Brustm, C., Hinterbichler, K.: Free box k scalar conformal field theory. In: Journal of high energy physics 2017.2 (2017). issn: 1029-8479. https://doi.org/10.1007/JHEP02(2017)066

4. De Lagrange, J.-L. In: Mem. Acad. Royale des Sciences et Belles-Lettres de Berlin 24, pp. 251-326 (1770)

5. Federbush, P.G., Johnson, K.A.: Uniqueness property of the twofold vacuum expectation value. In: Physical review 120.5, pp. 1926-1926 (1960). https://doi.org/10.1103/PhysRev.120.1926

6. Figueroa, H., Gracia-Bondia, J.M., Varilly, J.C.: Faa di bruno hopf algebras. In: arXiv:0508337 (visited on 10/14/2018) (2005)

7. Flajolet, P., Sedgewick, R.: Analytic combinatorics. OCLC: ocn244767782, p. 810. Cambridge University Press, Cambridge (2009). isbn: 978-0-521-89806-5 
8. Flume, R.: The invariance of the s-matrix under point transformations in renormalized perturbation theory. In: Communications in mathematical physics 40.1, pp. 49-54 (1975). issn: 0010-3616, 14320916. https://projecteuclid.org/euclid.cmp/1103860421 (visited on 01/20/2019)

9. Gracey, J.A., Simms, R.M.: Higher dimensional higher derivative phi4 theory. In: Physical Review D 96.2 (2017). issn: 2470-0010, 2470-0029. https://doi.org/10.1103/PhysRevD.96.025022. arXiv: 1705.06983

10. Haag, R.: On quantum field theories. In: Det Kongelige Danske Videnskabernes Selskab Matematiskfysiske Meddelelser 29.12, p. 19 (1955)

11. Heisenberg, W.: Die beobachtbaren Größen in der Theorie der Elementarteilchen. In: Zeitschrift ffn"ugr Physik 120.7, pp. 513-538 (1943). issn: 0044-3328. https://doi.org/10.1007/BF01329800

12. Henrici: An algebraic proof of the lagrange-buermann formula. In: Journal of mathemat- ical analysis and applications 8, pp. 218-224 (1964)

13. Jackson, D.M., Kempf, A., Morales, A.H.: A robust generalization of the legendre transform for QFT. In: Journal of Physics A: Mathematical and Theoretical 50.22, p. 225201 (2017). issn: 1751-8113, 1751-8121. https://doi.org/10.1088/1751-8121/aa6abb arXiv:1612.00462

14. Jost, J.: Properties of wightman functions. In: Caianiello, E.R. (ed.) Lectures on field theory and the many- body problem. Academic Press, New York (1961)

15. Klaczynski, L.: Haag's theorem in renormalised quantum field theories. In: arXiv:1602.00662. [hepth, physics:math-ph]. (visited on 11/14/2018). (2016)

16. Kreimer, D., Velenich, A.: Field diffeomorphisms and the algebraic structure of perturbative expansions. In: Lett. Math. Phys. 103, pp. 171-181 (2013). https://doi.org/10.1007/s11005-012-0589-y. arXiv:1204.3790 [hep-th]

17. Kreimer, D., Yeats, K.: Diffeomorphisms of quantum fields. In: Mathematical physics, analysis and geometry 20, 16, p. 16 (2017). https://doi.org/10.1007/s11040-017-9246-0. arXiv:1610.01837 [math$\mathrm{ph}]$

18. Lehmann, H., Symanzik, K., Zimmermann, W.: Zur formulierung quantisierter feldtheorien. In: Il Nuovo Cimento (1955-1965) 1.1, pp. 205-225 (1955). issn: 1827-6121. https://doi.org/10.1007/BF02731765

19. Mannheim, P.D.: Conformal gravity challenges string theory. In: arXiv:0707.2283 [gr-qc, physics:hep-th]. (visited on 01/10/2020) (2007)

20. Mannheim, P.D.: Solution to the ghost problem in fourth order derivative theories. In: Foundations of physics 37.4-5, pp. 532-571 (2007). issn: 0015-9018, 1572-9516. https://doi.org/10.1007/s10701007-9119-7. arXiv:0608154

21. Merlini, D., Sprugnoli, R., Verri, M.C.: Lagrange inversion: when and how. In: Acta applicandae mathematica 94.3 , pp. 233-249 (2006). issn: 1572-9036. https://doi.org/10.1007/s10440-006-9077-7

22. Nakanishi, N., Ojima, I.: Covariant operator formalism of gauge theories and quantum gravity. WORLD SCIENTIFIC, pp. 452 https://doi.org/10.1142/0362 (1990)

23. Omote, M.: Point canonical transformations and the path integral. In: Nuclear Physics B 120.2, pp. 325-332 (1977). issn: 0550-3213. https://doi.org/10.1016/0550-3213(77)90047-5

24. Pohlmeyer, K.: The jost-schroer theorem for zero-mass fields. In: Communications in mathematical physics 12.3, pp. 204-211 (1969). issn: 1432-0916. https://doi.org/10.1007/BF01661574

25. Salvio, A., Strumia, A.: Quantum mechanics of 4-derivative theories. In: The European Physical Journal C 76.4, p. 227 (2016). issn: 1434-6052. https://doi.org/10.1140/epjc/s10052-016-4079-8

26. Zee, A.: Einstein gravity emerging from quantum weyl gravity. In: Annals of Physics 151.2, pp. 431443 (1983). issn: 0003-4916. https://doi.org/10.1016/0003-4916(83)90286-5

Publisher's Note Springer Nature remains neutral with regard to jurisdictional claims in published maps and institutional affiliations. 